\title{
Crossing Network versus Dealer Market: Unique Equilibrium in the Allocation of Order Flow
}

Jutta Dönges*
Frank Heinemann** Tijmen R. Daniëls***

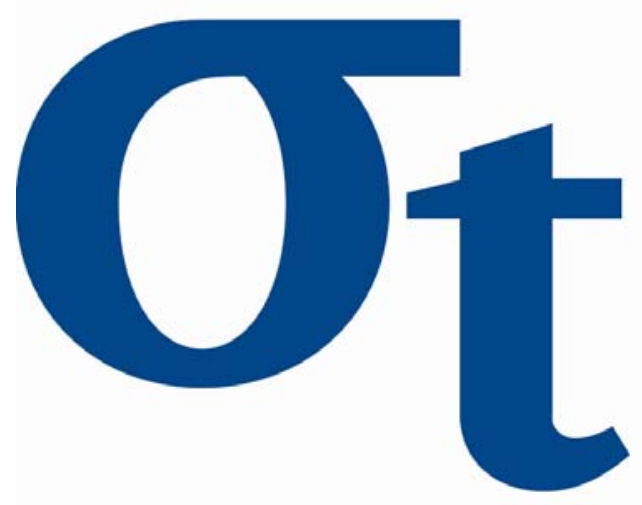

This research was supported by the Deutsche Forschungsgemeinschaft through the SFB 649 "Economic Risk".

http://sfb649. wiwi.hu-berlin.de ISSN 1860-5664 


\title{
CROSSING NETWORK VERSUS DEALER MARKET: UNIQUE EQUILIBRIUM IN THE ALLOCATION OF ORDER FLOW
}

\author{
JUTTA DÖNGES \\ SEB AB Frankfurt Branch \\ Ulmenstraße 23-25, 60325 Frankfurt am Main, Germany
}

FRANK HEINEMANN

Technische Universität Berlin

Institut für Volkswirtschaftslehre und Wirtschaftsrecht, Fachgebiet Makroökonomie Sekr. H52, Straße des 17. Juni 135, 10623 Berlin, Germany

\section{TIJMEN R. DANIËLS}

\author{
De Nederlandsche Bank N.V., Financial Stability Division \\ Postbus 98, 1000 AB Amsterdam, The Netherlands
}

\begin{abstract}
The allocation of order flow to alternative trading systems can be understood as a game with strategic substitutes between buyers on the same side of the market, as well as one of positive network externalities. We consider the allocation of order flow between a crossing network and a dealer market and show that small differences in traders' preferences generate a unique switching equilibrium, in which patient traders use the crossing network while impatient traders submit orders directly to the dealer market. Our model explains why assets with large turnovers and low price volatility are likely to be traded on crossing networks, while less liquid assets are traded on dealer markets.
\end{abstract}

KEY WorDs. Trading platform, order flow, strategic complements, strategic substitutes, global game JEL Codes. C62, G10, G20.

This is a substantially revised version of the working paper by the first two authors circulated under the same title. We thank Gerhard Illing, Oliver Kirchkamp, Philipp König, Stephen Morris, Ady Pauzner, Hyun Song Shin, Xavier Vives and Mark Wahrenburg, and participants at the Econometric Society European Meeting 2011 for stimulating discussions. Support from Deutsche Forschungsgemeinschaft (DFG) through SFB649 "economic risk" and a joint grant with ANR is gratefully acknowledged. Views expressed in this paper are not necessarily those of SEB or de Nederlandsche Bank. 


\section{Introduction}

Traders who do not require immediate execution of orders can reduce trading costs by searching for counterparties on alternative trading systems (ATS), instead of going to traditional dealer markets. New communication technologies and deregulatione.g. the EU's MiFID directive - have fuelled the development of such systems (see overviews by Degryse [7] or Stoll [28]). Some are sidelines of broker-dealers, and some are "dark pools" that aim to reveal as little information about trades as possible. Examples of opaque trading systems are crossing networks, which allow traders to enter orders that are crossed at a specific time at a price derived from another market. ${ }^{1}$ Offering lower commission, after-hours trading and anonymity, these systems direct order flow away from traditional exchanges, reducing transparency and potentially fragmenting liquidity. The development is of interest to economists, regulators, investors, and others who closely follow the emergence of new market structures.

A major question is how liquidity will be allocated and under which circumstances and for which sorts of assets, these alternative markets can emerge, co-exist with dealer markets or even replace them. It is not immediately obvious whether traders would keep on using traditional markets if there are cheaper alternatives. However, one barrier for adoption is that new venues must attract sufficient order flow to ensure a sufficiently high probability of order execution. When traders choose venues, strategic complements arise due to the liquidity externality of a deep market. As in other settings with strategic complements, this may lead to the existence of multiple equilibria. Previous theoretical research that has explored the issue of competition between trading venues has suffered from an indeterminacy due to the multiple equilibria, impeding policy implications. In some equilibria, alternative markets almost completely replace dealer markets, while in others they fail to attract sufficient liquidity to be viable.

In this paper, we investigate the allocation of order flow between a traditional dealer market (DM) and a crossing network $(\mathrm{CN})$. We contribute to the literature on the allocation of order flow by removing the multiplicity of equilibria that has plagued previous models. In our model, trading on a DM guarantees immediate order execution at bid and ask prices quoted by market makers. Trading on the electronic market is less expensive, as traders do not have to pay for an intermediary's services but only a small commission. However, the execution of an order submitted is uncertain because the number of buyers and sellers that are active in the market is ex ante unknown. The allocation of order flow can be understood as a game with strategic substitutes as well as strategic complements. Strategic substitutes exist because traders on the same side of a venue compete for orders. But if sufficiently many buyers and sellers coordinate to trade on a new venue, the probability of

\footnotetext{
${ }^{1}$ Examples of crossing networks are Instinet, POSIT, E-Crossnet and XETRA XXL. Degryse, Van Achter and Wuyts [10] provide an overview of recent market developments.
} 
order execution is high and expected payoff from trading on this market may exceed the payoff from trading on a dealer market. We show that the positive network externalities arising from overall market size dominate the strategic substitutes induced by competition of orders on the same side, so that one can apply the global game approach of Carlsson and van Damme [3] and Morris and Shin [22] to derive a monotonic switching equilibrium.

Heterogeneity is the driving force in our model. Since the two venues differ in features, they attract different kinds of traders. In our model's equilibrium, traders with a strong preference for immediate order execution trade at traditional DMs, while patient traders prefer submitting orders to a $\mathrm{CN}$, other things equal. Markets coexist when the CN is sufficiently liquid and there is sufficiently large heterogeneity of traders, so that some agents are patient and some are not. Traders whose liquidity preference is below a certain threshold then go to the CN. A unique such threshold exists, and it is increasing in the liquidity of the market, measured by the expected number of traders. This allows us to answer the question under which conditions a $\mathrm{CN}$ can coexist with a dealer market and conduct comparative statics.

These features of our model explain why assets with large turnovers and low price volatility are likely to be traded on crossing networks, while less liquid assets are traded on dealer markets, as empirical evidence also shows. Using a propriety data set on trading, Conrad et al. [5] find that CNs attract mostly stocks with large market capitalisation and high trading volume. Gresse [16] finds that less risky and more actively traded stocks are more likely to trade on a CN, based on LSE and Irish stock market data. Mao Yeh [20] studies NSYE and NASDAQ stocks and finds that the market share of crossing networks is higher for lower volatility stocks and higher volume. More generally, Theissen [29] reports evidence that market share of competing electronic trading venues increases with the trading volume of a stock and is negatively related to return volatility.

Market liquidity is a crucial parameter in our model. When liquidity is low, there is a substantial risk of mis-coordination by buyers and sellers. The CN becomes a viable trading venue only if it attracts sufficient liquidity to reduce the coordination risk. It is something that alternative trading systems have to deal with in reality. For crossing networks, this coordination risk is strengthened by their opaque character and potentially low order execution probabilities. Gresse [16] describes how in the eighties, a first attempt at establishing a European CN, ARIEL, failed to attract sufficient liquidity. She also documents probabilities of order execution on CNs as low as 2-4\%. Theissen [29] reports that XETRA, after expanding the set of traded stocks beyond an initial set, reduced its number of auctions due to lack of liquidity.

Several other papers study competition for order flow between a DM and an alternative market. These papers have one thing in common: there are multiple equilibria due to the presence of liquidity externalities. This holds in particular for 
the seminal paper by Hendershott and Mendelson [18], who study the impact of introducing a passive CN on competitive DMs and trading behaviour. The most plausible equilibria share the property that patient traders use the CN exclusively, traders with medium liquidity preferences use DMs when their order could not be matched at the CN, and traders with a strong liquidity preference go to the DM directly. Glosten [14] examines an idealised electronic limit order book and shows that it does not invite competition from other markets while other markets do. Parlour and Seppi [26] present a model of competition for order flow between different pairings of pure limit order markets and hybrid specialist/limit order markets. Viswanathan and Wang [31] model a traders' choice between a limit-order book, a DM, and a hybrid market structure of the two when traders differ in size and risk aversion. Chowdhry and Nanda [4] analyse how the ability of traders to choose the trading venue affects functioning and liquidity of markets in the presence of informational asymmetries and liquidity traders, who are not allowed to switch to another market. They show that the market with the largest number of liquidity traders attracts liquidity and informed traders, resulting in a concentration of trading in this market.

Our paper also complements research that has focused on competition between trading platforms, but not on the qualitative features that make platforms different from one another. These models give equilibrium predictions that are considerably less sharp. Pagano [25] studies a model of competition between markets with identical features, and shows that they cannot coexist when they charge the same transaction fee, but may coexist when their transaction fees differ. In this case, there may be either fragmentation or consolidation of trading, depending on the traders' initial expectations about other traders' decisions where to trade. Ellison and Fudenburg [11] study a two market model with buyers and sellers which, like ours, exhibits liquidity externalities as well as strategic substitutes between agents on the same side of the market. They argue that the latter, which they call traders' "market impact", may discourage traders from switching to the other market, creating a multitude of stable equilibria with unequal market sizes in which the two markets coexist. By contrast, our model predicts a unique split of trading activity. In our model, the liquidity externality and the market impact affects the CN, but not the DM.

Technically related to our model, Gehrig [13] analyses competition between an intermediary who offers guaranteed execution and a decentralised search market where heterogeneous traders meet randomly. Search is associated with a lower probability of order execution. Gehrig shows that traders with a low liquidity preference choose bilateral trading instead of trading with an intermediary. As in our model, the probability of order execution at one of the markets is limited, which is key for the existence of a critical liquidity preference that divides the customers of the two markets. However, in Gehrig's model, there is no uncertainty about the number of active buyers or sellers. Introducing a CN where all buyers and sellers 
can meet, would guarantee multiple equilibria and the execution probability at the $\mathrm{CN}$ would be either zero or one.

Also related is Degryse et al. [9], who analyse the welfare effects of reduced transparency in a setting with a CN and a DM. They use a sequential structure with a priori known number of traders who know their position in the sequence of arrivals. Under these assumptions, the equilibrium can be derived using backward induction, but this setting is qualitatively different from ours, in which the number of traders is ex ante unknown and they arrive simultaneously.

Finally, our paper makes a technical contribution to a growing literature that seeks to relax strict strategic complements assumptions that are typical in global games. Goldstein and Pauzner [15], Karp et al. [19] and Daniëls et al. [6] study global game models that exhibit both strategic complements and substitutes. In these models, actions are complements in some region of the action space and substitutes in others. By contrast, in our model, actions are complements for some traders, and substitutes for others.

Our paper is organised as follows. In Section 2, we develop a benchmark model in which positive network externalities arise from the probability of order execution at a CN, illustrating the problem of equilibrium multiplicity. In Section 3 we introduce heterogeneity in liquidity preferences and prove that this leads to a unique switching equilibrium. The uniqueness of the switching equilibrium allows us to do comparative statics. In Section 4, we present welfare results based on our model's equilibrium. In Section 5, we present robustness results, discussing the possible relaxation of some of the simplifying assumptions of our model. Section 6 concludes.

\section{Traders and Markets: A Benchmark Model}

Traders choose market venues depending on transaction costs and liquidity, amongst other things. As more traders direct their orders to a particular venue, liquidity increases. This, in turn, attracts even more traders - there are positive network externalities. Because of this feature, the choice of a market venue may be understood as a coordination game with strategic complements: the difference in expected payoffs from directing an order to one venue versus another increases in the relative size of the venue.

This section develops a benchmark model in which traders choose to trade either at a dealer market or at a crossing network. Our benchmark model is a reduced form one-shot game, in which unexecuted orders leave the trader with some disutility. This disutility may arise from traders' impatience or urgency to trade or from the risk associated with an asset's price volatility. Positive network externalities arise from the fact that the probability of order execution at the crossing network is increasing in the liquidity directed to this network. As is typical for coordination 
games with strategic complements, our benchmark model has multiple equilibria. In our benchmark model, trading costs and asset prices are exogenous. Changing these features typically strengthens the presence of strategic complements, as we will explain in Section 5.

\section{$\S 2.1$. The Model}

Consider an asset that trades at a dealer market (DM) and at a crossing network $(\mathrm{CN})$. A finite, random number of traders is active at each side of the market; the number of buyers is $N_{b}$, the number of sellers is $N_{s}$. We assume that $N_{b}$ and $N_{s}$ are independently and identically distributed, and that with strictly positive probability $N_{b} \neq N_{s}$. Each trader decides to trade at the DM or the CN.

In the DM, traders trade with market makers who set bid and ask prices at which they are willing to buy or sell the asset. We normalise the mid-point of bid and ask price to zero, so that traders can buy the asset at price $t_{\mathrm{DM}}$ and sell at $-t_{\mathrm{DM}}$, where $t_{\mathrm{DM}}$ is half of the bid-ask spread.

The CN offers transactional services without any intervention by an intermediary. Orders can be submitted to the CN as market orders and are executed at the midpoint between the bid and ask price observed on the DM, i.e. zero. Traders who have submitted to the $\mathrm{CN}$ run the risk of their orders not being executed. The numbers of buyers and sellers who place their orders in the $\mathrm{CN}$, denoted by $n_{b}$ and $n_{s}$, determine the probabilities with which orders are executed. If there is an imbalance of orders on the two sides of the $\mathrm{CN}$, the excess side is rationed stochastically. Orders on the excess side are then randomly selected to match orders on the short side. The execution probability of a buy order in the $\mathrm{CN}$ is therefore

$$
\pi_{b}=\min \left\{1, n_{s} / n_{b}\right\}
$$

and the execution probability of a sell order is

$$
\pi_{s}=\min \left\{1, n_{b} / n_{s}\right\} .
$$

If an order is executed in the $\mathrm{CN}$, the trader pays a small fee $t_{\mathrm{CN}}<t_{\mathrm{DM}} \cdot{ }^{2}$

Since the total numbers of buyers $N_{b}$ and of sellers $N_{s}$ are unknown to traders when they submit their orders, they always face some uncertainty about whether or not an order at the $\mathrm{CN}$ will be executed. Unexecuted orders may be resubmitted to the market in the next period or passed to a dealer. Either way, trades are executed with delay and possibly at a different price. We assume that this leaves the trader with some disutility $\theta>t_{\mathrm{CN}}$. We refer to $\theta$ as the trader's liquidity preference: the higher $\theta$, the more the trader values a liquid market, where orders are quickly executed.

\footnotetext{
${ }^{2}$ This is a feature of some ATS in reality: POSIT, for instance, allows submission of orders free of charge, charging a fee when orders are executed. Including a submission fee does not change the results, as a fee for unexecuted orders can be subsumed in $\theta$.
} 
Let $\alpha_{s}$ be the proportion of sellers that go to the $\mathrm{CN}$, and $\alpha_{b}$ the proportion of buyers. Given these proportions and a random process that determines the number of active buyers and sellers, the expected probabilities of order execution are well defined. Denote the execution probability of a buy order on the CN by $\pi\left(\alpha_{b}, \alpha_{s}\right)$. By symmetry, the execution probability of a sell order is $\pi\left(\alpha_{s}, \alpha_{b}\right)$. For a buyer the expected payoff from going to the $\mathrm{CN}$ instead of going to the DM is therefore

$$
U\left(\theta, \alpha_{b}, \alpha_{s}\right)=\left(\theta-t_{\mathrm{CN}}\right) \pi\left(\alpha_{b}, \alpha_{s}\right)-\left(\theta-t_{\mathrm{DM}}\right) .
$$

By symmetry, for a seller the expected payoff from going to the $\mathrm{CN}$ instead of going to the DM is

$$
U\left(\theta, \alpha_{s}, \alpha_{b}\right)=\left(\theta-t_{\mathrm{CN}}\right) \pi\left(\alpha_{s}, \alpha_{b}\right)-\left(\theta-t_{\mathrm{DM}}\right)
$$

\section{§2.2. Geometric Distribution of Arrival}

To gain further intuition, we may adopt a specific functional form for the function $\pi$. Consider the geometric distribution adopted by Hendershott and Mendelson (2000), which arises as follows. With probability $p$, a first buyer comes to the market. If, and only if, a first buyer comes to the market, a second buyer comes again with probability $p$, and so on. The probability of having at least $n$ buyers is thus $p^{n}$, and the total number of buyers $N_{b}$ follows a geometric distribution with expectation value $\mathbf{E}\left[N_{b}\right]=p /(1-p)=: \lambda$. Sellers come according to the same process. This parametrisation has the advantage that $\lambda$ is a measure of the overall thickness of the market. The following result shows how market thickness affects the probability of order execution.

Lemma 1. If $N_{b}$ and $N_{s}$ are independently drawn from a geometric distribution with $\mathbf{E}\left[N_{b}\right]=\mathbf{E}\left[N_{s}\right]=\lambda$, the execution probability of a buy order on the $C N$ is

$$
\pi\left(\alpha_{b}, \alpha_{s}\right)=\frac{\alpha_{s}}{\alpha_{b}} \ln \left(1+\frac{\alpha_{b} \lambda}{1+\alpha_{s} \lambda}\right)
$$

and the execution probability of a sell-order is $\pi\left(\alpha_{s}, \alpha_{b}\right)$ by symmetry.

The proof of this lemma is in the appendix. Hendershott and Mendelson [18, Proposition 3, p. 2081] prove the special case where $\alpha_{b}=\alpha_{s}$.

\section{$\S 2.3$. Equilibrium}

We assume traders are risk neutral and maximise expected payoff. In a Nash equilibrium, traders go to the market with the highest expected payoff. Thus,

$$
\alpha_{b}=\left\{\begin{array}{ll}
1 & \text { if } U\left(\theta, \alpha_{b}, \alpha_{s}\right)>0 \\
0 & \text { if } U\left(\theta, \alpha_{b}, \alpha_{s}\right)<0
\end{array} \quad \text { and } \quad \alpha_{s}= \begin{cases}1 & \text { if } U\left(\theta, \alpha_{s}, \alpha_{b}\right)>0 \\
0 & \text { if } U\left(\theta, \alpha_{s}, \alpha_{b}\right)<0\end{cases}\right.
$$


We now show that, in any Nash equilibrium, the same proportions of buyers and sellers submit orders to the CN. Associated with each Nash equilibrium is therefore a market share $\alpha^{*}$ for the $\mathrm{CN}$.

Lemma 2. In any Nash equilibrium of the benchmark model, $\alpha_{b}=\alpha_{s}=: \alpha^{*}$.

Proof. Suppose that different proportions of buyers and sellers submit orders to the CN, for example, suppose $\alpha_{s}<\alpha_{b}$. Then their probabilities of order execution differ as well. In fact, we have $\pi\left(\alpha_{b}, \alpha_{s}\right)<\pi\left(\alpha_{s}, \alpha_{b}\right)$, so that

$$
U\left(\theta, \alpha_{b}, \alpha_{s}\right)<U\left(\theta, \alpha_{s}, \alpha_{b}\right) .
$$

Moreover, $\alpha_{s}<\alpha_{b}$ implies $\alpha_{b}>0$ and $\alpha_{s}<1$. Now, in equilibrium traders would go to the market with the highest payoff, so that $\alpha_{b}>0$ and $\alpha_{s}<1$ together would imply $U\left(\theta, \alpha_{b}, \alpha_{s}\right) \geq 0$ and $U\left(\theta, \alpha_{s}, \alpha_{b}\right) \leq 0$. But that contradicts inequality (1).

Having established that the same proportion $\alpha^{*}$ of buyers and sellers submit orders to the $\mathrm{CN}$, we can turn to the question what determines the CN's market share. As it is ex ante uncertain which side of the market will be the long one, all traders face some risk of being rationed if they go to the CN. After all, the numbers of buyers and sellers of the asset are drawn independently, and thus the buy and sell sides of the market are rarely exactly balanced. Therefore, the ex-ante probability of order execution on the $\mathrm{CN}$ is bounded away from 1, even if all potential traders decide to go to the $\mathrm{CN}$.

For the geometric distribution of the number of traders, and market share $\alpha$, the probability of order execution is

$$
\pi(\alpha)=\ln \left(1+\frac{\alpha \lambda}{1+\alpha \lambda}\right),
$$

where $\pi(\alpha)$ abbreviates $\pi(\alpha, \alpha)$, the probability of order execution given market share $\alpha$ with the equality of the function's two arguments implicitly understood. The function $\pi(\alpha)$ is increasing in $\alpha$ up to

$$
\bar{\pi}=\pi(1)=\ln \left(1+\frac{\lambda}{1+\lambda}\right)<0.7 \text {. }
$$

For $\lambda \rightarrow \infty$, the probability of order execution converges to $\ln (2) \approx 0.693$, so that $\bar{\pi}$ remains bounded away from 1 . Thus, the $\mathrm{CN}$ is always riskier than the DM, at which order execution is guaranteed.

If the disutility $\theta$ from an unexecuted order is large, even a small risk of an order not being executed in the $\mathrm{CN}$ outweighs the difference in fees. The tipping point is given by the $\tilde{\theta}$ for which both market venues promise the same expected payoff, provided that all traders go to the $\mathrm{CN}$ :

$$
\tilde{\theta}=\frac{t_{\mathrm{DM}}-\bar{\pi} t_{\mathrm{CN}}}{\frac{1-\bar{\pi}}{8}} .
$$




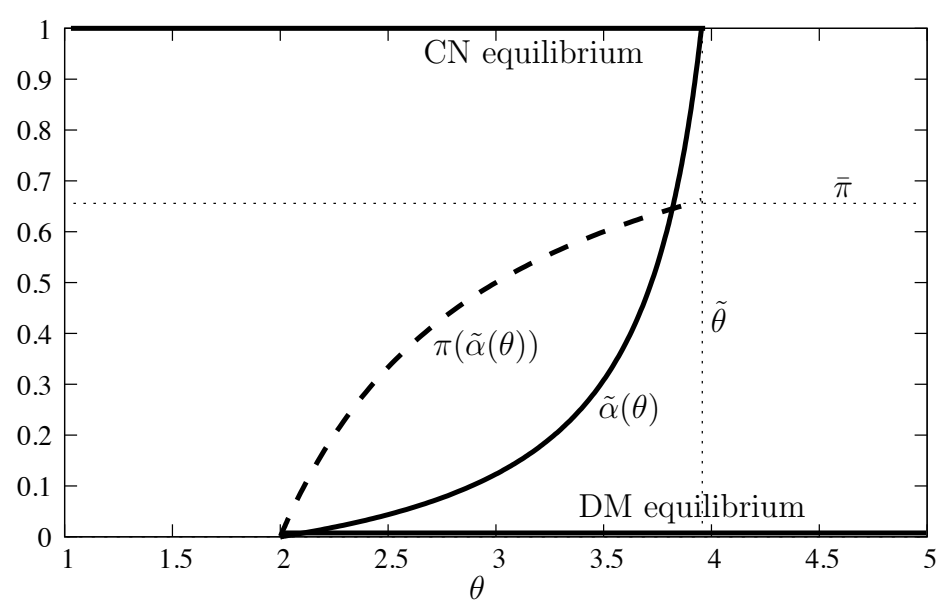

For $t_{\mathrm{CN}}=1, t_{\mathrm{DM}}=2$ and $\lambda=15$, the upper limit of the multiplicity region is $\tilde{\theta}=3.953$, while $\bar{\pi}=0.6614$.

Figure 1. Nash Equilibria of the benchmark model.

For liquidity preference $\theta>\tilde{\theta}$, the payoff on the DM exceeds the expected payoff in the $\mathrm{CN}$, even if all others traders coordinate to use the CN. In this case, submitting orders to the DM is a dominant strategy and the market share of the $\mathrm{CN}$ is $\alpha^{*}=0$. Note that, under the geometric distribution, if $\lambda \rightarrow \infty$, the tipping point $\tilde{\theta}$ converges to the finite number $\left(t_{\mathrm{DM}}-\ln (2) t_{\mathrm{CN}}\right) /(1-\ln (2))$ and not to infinity. Thus, even when the market is deep, a sufficiently large liquidity preference will induce traders to trade on the DM. For $t_{\mathrm{CN}}<\theta<t_{\mathrm{DM}}$, traders lose from trading on the DM and have positive expected payoffs in the $\mathrm{CN}$. In this case, trading in the $\mathrm{CN}$ is a dominant strategy and $\alpha^{*}=1$.

For intermediate values, $t_{\mathrm{DM}} \leq \theta \leq \tilde{\theta}$, there are three possible Nash equilibria. In one Nash equilibrium, all traders go to the DM. A single trader cannot gain by switching to the $\mathrm{CN}$, because without a trading partner her order would not be executed. In another Nash equilibrium, all traders go to the CN. If the CN attracts sufficient liquidity, no trader has an incentive to leave the CN. Of these two equilibria, coordination on the $\mathrm{CN}$ is the Pareto efficient equilibrium, because it maximises expected payoff: $\bar{\pi}\left(\theta-t_{\mathrm{CN}}\right) \geq\left(\theta-t_{\mathrm{DM}}\right)$, where the inequality follows from (2).

In addition to these pure Nash equilibria, there is a mixed equilibrium, in which both markets co-exist. In this equilibrium, a proportion $\tilde{\alpha}(\theta) \in(0,1)$ of traders go to the CN. Given the market share of the $\mathrm{CN}$, the execution probability on the $\mathrm{CN}$ equalises expected payoffs at both markets, so no trader wants to switch. Under the geometric selection process, the market share of the $\mathrm{CN}$ in the mixed equilibrium equals

$$
\tilde{\alpha}(\theta)=\frac{1}{\lambda} \cdot\left[\exp \left(\frac{\theta-t_{\mathrm{DM}}}{\theta-t_{\mathrm{CN}}}\right)-1\right] /\left[2-\exp \left(\frac{\theta-t_{\mathrm{DM}}}{\theta-t_{\mathrm{CN}}}\right)\right] .
$$

The next proposition summarises the possibilities. Figure 1 plots the equilibria of the benchmark model with geometric distribution. 
Proposition 3. In any Nash equilibrium of the benchmark model, one of the following three situations holds:

$$
\begin{aligned}
& \theta \in\left(t_{\mathrm{CN}}, t_{\mathrm{DM}}\right) \quad \text { and } \quad \alpha_{b}=\alpha_{s}=\alpha^{*}=1 \text {, } \\
& \theta \in\left[t_{\mathrm{DM}}, \tilde{\theta}\right] \quad \text { and } \quad \alpha_{b}=\alpha_{s}=\alpha^{*} \in\{0, \tilde{\alpha}(\theta), 1\} \text {, } \\
& \theta \in(\tilde{\theta}, \infty) \quad \text { and } \quad \alpha_{b}=\alpha_{s}=\alpha^{*}=1 .
\end{aligned}
$$

The CN's market share in the mixed equilibrium, $\tilde{\alpha}(\theta)$, increases from 0 to 1 on the interval $\left[t_{\mathrm{DM}}, \tilde{\theta}\right]$.

One may assume a selection process that is different from the geometric process. For instance, the number of buyers $N_{b}$ and sellers $N_{s}$ may follow a Poisson distribution, as in Myerson [24]; or the stochastic process may be chosen to reflect the propriety rationing algorithm used by a particular crossing network (Degryse et al. [10] provide an overview of algorithms used in practice). Lemma 2 and Proposition 3 and most other results in the paper carry over if we impose some general properties on the order execution probability $\pi(\cdot)$ at $\mathrm{CN}$, all of which hold if traders are drawn according to a geometric process.

$$
\begin{aligned}
& \pi\left(\alpha_{b}, \alpha_{s}\right) \text { is continuous and bounded away from } 1 ; \\
& \text { if } \alpha_{s}<\alpha_{b} \text { then } \pi\left(\alpha_{b}, \alpha_{s}\right)<\pi\left(\alpha_{s}, \alpha_{b}\right) ; \\
& \pi(\alpha) \text { is an increasing function of } \alpha ; \\
& \pi\left(\alpha_{b}, \alpha_{s}\right)=0 \text { if and only if } \alpha_{s}=0 .
\end{aligned}
$$

Assumption (3) guarantees there is always some uncertainty about order execution at the $\mathrm{CN}$; (4) requires that, if the proportion of buyers that go to the $\mathrm{CN}$ exceeds the number of sellers, the expected probability of execution of a buy order is smaller than that of a sell order; (5) says that the probability of execution of an order is increasing in the market share of the $\mathrm{CN}$; and (6) that the probability of execution of a buy order on the CN is zero if and only if there are no sellers that go to the CN. No other properties were used for Lemma 2 and Proposition 3.

\section{A Model with Heterogeneous Liquidity Preferences}

Which of the equilibria of the benchmark game should one expect in situations of inter-market competition? In this section, we show that the multiplicity of equilibria of the benchmark game hinges on the assumption of a liquidity preference $\theta$ that is common to all traders. If liquidity preferences differ across traders, the market share $\alpha^{*}$ of the CN may be uniquely determined. 


\section{§3.1. Private Liquidity Preferences}

Suppose each trader $i$ has a private liquidity preference $\theta_{i}$. We assume that each $\theta_{i}$ is the sum of two components: a common part $\theta$, which may be interpreted as an aggregate liquidity shock, and a private, idiosyncratic liquidity shock. More precisely

$$
\theta_{i}=\theta+\nu u_{i}
$$

where $\nu$ is a scale parameter and each $u_{i}$ is drawn independently from a probability distribution with symmetric density function $f$ and with compact support $\left[-\frac{1}{2},+\frac{1}{2}\right]$. The distribution $f$ and scale factor $\nu$ are common knowledge, and each trader knows her own preference $\theta_{i}$. However, traders know the aggregate liquidity shock only up to their private idiosyncratic shock $u_{i}$. We assume traders' prior with respect to the aggregate shock is uniform on the interval $\left[\theta_{L}, \theta_{U}\right]$ which contains $t_{\mathrm{DM}}$ and $\tilde{\theta}$ in its interior: $t_{\mathrm{CN}}+\frac{\nu}{2} \leq \theta_{L} \leq t_{\mathrm{DM}}-\frac{\nu}{2}$ and $\tilde{\theta}+\frac{\nu}{2} \leq \theta_{U}$. Finally, traders come to the market according to a random process satisfying the properties described in Section 2.

This setup is an example of the "private value" global game approach in the spirit of Frankel, Morris and Pauzner [12] and Morris and Shin [23], but with one important difference. In global games, decisions of agents are typically strategic complements. In our model, the decisions of buyers are complements to those of sellers (going to the same markets increases the probability of order execution) but traders on the same side of the market compete with each other for being matched in the $\mathrm{CN}$ and so their choices are strategic substitutes instead of complements. As we will show, the global-game approach may be generalised to this situation, establishing the existence of a unique switching equilibrium for our model.

\section{§3.2. Switching Equilibrium}

Let $a_{s}\left(\theta_{i}\right)$ denote the proportion of sellers who submit to the $\mathrm{CN}$ when their private liquidity preference is $\theta_{i}$. The proportion of them who go to the $\mathrm{CN}$ when the aggregate shock is equal to $\theta$ is now given by

$$
\alpha_{s}\left(\theta, a_{s}\right)=\frac{1}{\nu} \int_{\theta_{i}} a_{s}\left(\theta_{i}\right) f\left(\frac{1}{\nu}\left(\theta_{i}-\theta\right)\right) \mathrm{d} \theta_{i} .
$$

The proportion of buyers who go to the $\mathrm{CN}$ when the aggregate liquidity shock is $\theta$, denoted $\alpha_{b}\left(\theta, a_{b}\right)$, is defined analogously. The relative payoff of going to the CN for a buyer with liquidity preference $\theta_{i}$ is now:

$$
U\left(\theta_{i}, \alpha_{b}, \alpha_{s}\right)=\left(\theta_{i}-t_{\mathrm{CN}}\right) \pi^{e}\left(\theta_{i}, \alpha_{b}, \alpha_{s}\right)-\left(\theta_{i}-t_{\mathrm{DM}}\right),
$$

where the expected probability of order execution $\pi^{e}$ is given by

$$
\pi^{e}\left(\theta_{i}, \alpha_{b}, \alpha_{s}\right)=\frac{1}{\nu} \int_{\theta} \pi\left(\alpha_{b}\left(\theta, a_{b}\right), \alpha_{s}\left(\theta, a_{s}\right)\right) f\left(\frac{1}{\nu}\left(\theta-\theta_{i}\right)\right) \mathrm{d} \theta .
$$


A switching equilibrium is a profile of strategies for sellers and buyers characterised by two thresholds $\theta_{b}^{*}, \theta_{s}^{*}$, such that a buyer goes the $\mathrm{CN}$ if and only if her private liquidity preference $\theta_{i}$ is smaller than the threshold value $\theta_{b}^{*}$ and, likewise, a seller goes to the $\mathrm{CN}$ if and only if her private liquidity preference $\theta_{i}$ is smaller than $\theta_{s}^{*}$, and such that no buyer or seller wants do deviate unilaterally.

Recall from Lemma 2 that all equilibria of the benchmark game are symmetric, in the sense that the same proportions of buyers and sellers submit orders to the CN. The key observation for our argument is that a modified version of this principle must also be true in the private value game. More precisely, let us call a switching equilibrium symmetric if buyers and sellers use the same thresholds, i.e. we have $\theta_{b}^{*}=\theta_{s}^{*}$. In this case, for each $\theta$, the proportion of buyers and sellers that go to the same market is clearly identical. We now prove the following result:

Lemma 4. Any switching equilibrium is symmetric.

The logic is almost identical to that of Lemma 2. In equilibrium, buyers and sellers must face the same probability of order execution, and this can happen only if the proportions of buyers and sellers entering the $\mathrm{CN}$ are identical.

Proof. Suppose, without loss of generality, that there exists a switching equilibrium such that $\theta_{s}^{*}<\theta_{b}^{*}$. Then for each $\theta, \alpha_{s}(\theta) \leq \alpha_{b}(\theta)$, and this inequality holds strictly in some neighbourhood $\mathcal{N}$ of $\theta_{b}^{*}$. By property (4), this implies $\pi\left(\alpha_{b}(\theta), \alpha_{s}(\theta)\right) \leq$ $\pi\left(\alpha_{s}(\theta), \alpha_{b}(\theta)\right)$, where the inequality again holds strictly for $\theta \in \mathcal{N}$. But then $\pi^{e}\left(\theta_{b}^{*}, \alpha_{b}, \alpha_{s}\right) \leq \pi^{e}\left(\theta_{b}^{*}, \alpha_{s}, \alpha_{b}\right)$, and hence $U\left(\theta_{b}^{*}, \alpha_{b}, \alpha_{s}\right)<U\left(\theta_{b}^{*}, \alpha_{s}, \alpha_{b}\right)$.

In a switching equilibrium, buyers with liquidity preferences smaller than $\theta_{b}^{*}$ must weakly prefer the $\mathrm{CN}$, while those with liquidity preferences greater than $\theta_{b}^{*}$ must weakly prefer the DM. Since expression (7) is continuous, this implies that buyers with liquidity preference $\theta_{b}^{*}$ are indifferent, so we have

$$
0=U\left(\theta_{b}^{*}, \alpha_{b}, \alpha_{s}\right)<U\left(\theta_{b}^{*}, \alpha_{s}, \alpha_{b}\right),
$$

i.e. sellers strictly prefer the $\mathrm{CN}$ when their liquidity preference is $\theta_{b}^{*}$. But in a switching equilibrium all sellers with liquidity preferences exceeding $\theta_{s}^{*}$ must weakly prefer the DM, so this contradicts that $\theta_{s}^{*}<\theta_{b}^{*}$ can be thresholds in a switching equilibrium.

As a consequence of Lemma 4 , a market share $\alpha^{*}$ for the $\mathrm{CN}$ is associated with each $\theta$. It implies that traders' decisions are complementary, even though the model does not satisfy strategic complements. After all, the expected payoff for trader $i$ of going to the $\mathrm{CN}$ instead of the DM in expression (7) is increasing in the expected probability of order execution in the $\mathrm{CN}$. By property (5), for given $\theta$ this probability is increasing in the amount of traders who direct their orders to the CN. Thus, for each $\theta$, the expected payoff of going to the $\mathrm{CN}$ is increasing in the market share of the CN. For a fixed switching threshold $\theta^{*}:=\theta_{b}^{*}=\theta_{s}^{*}$, the market share of the CN is 
decreasing in $\theta$ and then equation (8) implies that the expected payoff of going to the $\mathrm{CN}$ is decreasing in the trader's own liquidity preference $\theta_{i}$.

We will now solve the game using arguments that are standard in the literature on global games. Define the monotonic strategy:

$$
I_{x}\left(\theta_{i}\right):=\left\{\begin{array}{ll}
1 & \text { if } \theta_{i} \leq x, \\
0 & \text { if } \theta_{i}>x,
\end{array} \quad x \in \mathbf{R} .\right.
$$

A trader that uses this strategy goes to the $\mathrm{CN}$ if and only if her private liquidity preference $\theta_{i}$ is smaller than $x$. Since $U\left(\theta_{i}, I_{x}, I_{x}\right)$ is continuous and, for fixed $x$, decreasing in its first argument, a switching strategy around $\theta^{*}$ is an equilibrium if and only if $U\left(\theta^{*}, I_{\theta^{*}}, I_{\theta^{*}}\right)=0$, viz. the trader with the critical liquidity preference $\theta^{*}$ is indifferent.

Recall that when $\theta_{i} \leq t_{\mathrm{DM}}$, the payoff in the CN exceeds the payoff in the DM, even if all other traders coordinate to use the CN. Similarly, when $\theta_{i} \geq \tilde{\theta}$, the payoff of the DM always exceeds that of the CN. By the intermediate value theorem, there is $x$ such that $U\left(x, I_{x}, I_{x}\right)=0$, which guarantees the existence of a switching equilibrium. If $\mathrm{d} U\left(x, I_{x}, I_{x}\right) / \mathrm{d} x$ is strictly negative for each solution of $U\left(x, I_{x}, I_{x}\right)=0$, then the switching equilibrium is necessarily unique.

We may therefore conclude our argument by showing that $\mathrm{d} U\left(x, I_{x}, I_{x}\right) / \mathrm{d} x<0$. The partial derivative of $U$ with respect to its first argument is strictly negative: an increase in $x$ increases expected return in the $\mathrm{CN}$ at a marginal rate that equals the execution probability of order execution $\pi^{e}<1$, while expected returns on the DM rise at a marginal rate of 1 .

As for its other two arguments, an increase in the switching point up to which traders use the $\mathrm{CN}$ affects the probabilities of order execution at any given fixed $\theta_{i}$. However, the key observation is: if buyers and sellers follow the strategy $I_{x}$, the probability of order execution for the traders that have the critical liquidity preference $x$ must always be the same. The reason, pointed out by Morris and Shin (2003), is that such a trader does not know whether she has a relatively high or a relatively low liquidity preference since other traders' liquidity preferences are independent of her own, conditional on the aggregate liquidity shock. Indeed, Morris and Shin showed that, conditional on having the threshold liquidity preference $x$, a trader's belief about the proportion of other traders who have a value of immediacy below the threshold must be uniform on $[0,1]$. Therefore an increase in $x$ unambiguously lowers the expected payoff $U\left(x, I_{x}, I_{x}\right)$ at a rate $1-\pi^{e}$. There indeed exists a unique solution to the equation $U\left(x, I_{x}, I_{x}\right)=0$ and the next result follows immediately.

Proposition 5. There is a unique critical liquidity preference $\theta^{*}$, such that in any switching equilibrium, traders with private liquidity preferences $\theta_{i}$ smaller $\theta^{*}$ trade at the $C N$, and those with liquidity preferences $\theta_{i}$ greater than $\theta^{*}$ trade at the DM. It is 


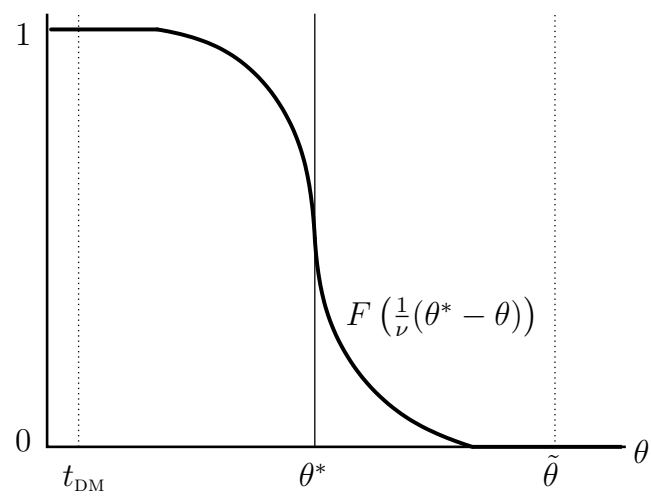

FiguRE 2. Market share of the CN with heterogeneous preferences given by

$$
\theta^{*}=\frac{t_{\mathrm{DM}}-\pi^{*} t_{\mathrm{CN}}}{1-\pi^{*}}
$$

where $\pi^{*}=\int_{0}^{1} \pi(\alpha) \mathrm{d} \alpha$ is the expected probability of order execution for those traders whose idiosyncratic liquidity preferences $\theta_{i}$ exactly equal $\theta^{*}$.

Note that $\theta^{*}$ and $\pi^{*}$ do not depend on the distribution of signals $f$ or scale factor $\nu$. For the geometric process, $\pi^{*}$ is equal to $\bar{\pi}-\frac{1}{2 \lambda} \ln \left(\frac{(1+\lambda)^{2}}{1+2 \lambda}\right)<\bar{\pi}$.

\section{§3.3. Equilibrium Implications}

In equilibrium, each trader weighs the expected payoff from trading at the CN against the certain payoff from trading at the DM. At the switching threshold $\theta^{*}$, these are exactly equal. In case the $\mathrm{CN}$ captures part of the market, agents that have low private liquidity preferences choose the $\mathrm{CN}$ and agents that have high private liquidity preferences choose the DM. For an asset with average liquidity preference $\theta$, the market share of the $\mathrm{CN}$ is $F\left((1 / \nu)\left(\theta^{*}-\theta\right)\right)$, where $F$ is the cumulative distribution of the idiosyncratic component in traders' liquidity preferences. If $\theta<\theta^{*}-\nu / 2$, all traders have liquidity preferences smaller than $\theta^{*}$ and choose to trade using the CN. If $\theta>\theta^{*}+\nu / 2$, all traders have preferences greater than $\theta^{*}$ and trade on the DM. For intermediate values of $\theta$, the market share of the $\mathrm{CN}$ increases from 0 to 1 - see Figure 2.

The uniqueness of the equilibrium allows us to do comparative statics.

Corollary 6. The critical threshold $\theta^{*}$ is increasing in $t_{\mathrm{DM}}$ and decreasing in $t_{\mathrm{CN}}$.

This may be shown by differentiating equation (10). Thus, the CN and DM influence their market share by setting the transaction costs appropriately.

The fact that trading venues attract different kinds of traders in the competition for order flow explains a number of trends in which venues try to reduce the costs of specific groups of traders. The fact that traders with high liquidity preferences are more likely to trade on the CN draws liquidity from the DM. After all, these are the 
traders that are most likely to post limit orders, providing liquidity to the market. Nowadays, nearly all exchanges subsidise such liquidity providers.

Conversely, in 2009 the trading exchange BATS started to pay a subsidy to liquidity takers who opt into routing their orders through BATS' ATS partners [1]. Our model allows us to see why. If an order can be matched on an ATS, it may be removed from BATS' order book, improving the ratio of liquidity providers to liquidity takers, and thus liquidity, on the exchange. At the same time, an ATS benefits if liquidity takers are encouraged to route their orders through it. Because of the existence of network externalities, more orders on the $\mathrm{CN}$ increases expected payoff also for other users of the CN, which makes the platform more attractive. Since individual traders do not take this externality into account, it may make sense to provide a subsidy. Alternatively, an ATS may compete for trades by offering services that are not available on traditional exchanges, such as after hour-trading or anonymity.

The other key parameter that influences the equilibrium threshold is market liquidity. To study its effects, we now turn back to the geometric distribution to investigate the effect of the market thickness parameter $\lambda$ on equilibrium. Note that $\pi(\alpha)$ is increasing in $\lambda$ for all $\alpha$, so that $\pi^{*}$ also increases in $\lambda$. Thus, from equation (10) we see:

Corollary 7. The critical threshold $\theta^{*}$ is increasing in $\lambda$.

This tells us that at CNs assets are traded for which the expected turnover is large. But, even for assets with the highest turnover, the disutility of order execution (and hence, asset features leading to high average liquidity preferences, such as volatility) is an important criterion for the allocation of order flow. This property of the equilibrium limits the potentials for a $\mathrm{CN}$ to compete with a DM: if intervals before crossings are extended to collect a larger number of orders (which raises $\lambda$ ), the probability of order execution increases, which makes the CN more attractive. But, the delay in the execution also increases expected costs. This limitation provides a trade-off to any CN. In reality, CNs such as POSIT have reduced the length of trading intervals only as overall liquidity has improved.

\section{§3.4. Relation to standard global games results}

The main insight to be gained from the model with heterogeneous liquidity preferences is that some uncertainty about other traders' private liquidity preferences are sufficient to establish a unique switching equilibrium. Our argument requires uncertainty about a trader's own position in the distribution of preferences amongst the traders who are active in the market. While this assumption is key in global games, it is also more realistic than assuming common knowledge of the distribution of preferences amongst active traders. By contrast, the common knowledge assumption is responsible for 
multiplicity of equilibria in the benchmark model. This mirrors similar findings in the broader global games literature.

To conclude this section, we further clarify the relation between our results and standard results on global games, which apply to games with strategic complements. Let us assume for the moment that buyers and sellers always use the same strategy, so that $a_{b}\left(\theta_{i}\right)=a_{s}\left(\theta_{i}\right):=a\left(\theta_{i}\right)$ for all $\theta_{i}$. Thus, equal proportions of buyers and sellers go to the $\mathrm{CN}$ when their liquidity preference is $\theta_{i}$. Given equal distributions of liquidity preferences among buyers and sellers, the proportions of agents that go to the $\mathrm{CN}$ when the true state is $\theta$ are also the same.

Now consider a modified game, in which agents choose to go to the $\mathrm{CN}$ or the DM before they learn whether they will want to buy or sell the asset, so that the strategies of buyers and sellers are indeed necessarily identical. The payoffs of going to the DM instead of the CN are then given by:

$$
U\left(\theta_{i}, \alpha\right)=\left(\theta_{i}-t_{\mathrm{CN}}\right) \pi^{e}\left(\theta_{i}, \alpha, \alpha\right)-\left(\theta_{i}-t_{\mathrm{DM}}\right) .
$$

Since $\pi(\alpha)$ is increasing in $\alpha$, it is straightforward to verify that this modified game satisfies the usual strategic complements assumption from the global games literature. Therefore, by standard results (e.g. Frankel, Morris and Pauzner [12]), this game has a unique switching equilibrium.

We claim that $a$ is an equilibrium of the modified game if and only if it is a symmetric equilibrium of our original game. To see this, note that $a$ is an equilibrium in the modified game if and only if $a\left(\theta_{i}\right)>0 \Leftrightarrow U_{k}\left(\theta_{i}, \alpha\right) \geq 0$ and $a\left(\theta_{i}\right)<1 \Leftrightarrow U_{k}\left(\theta_{i}, \alpha\right) \leq 0$ hold for each $\theta_{i}$. But this is precisely the necessary and sufficient condition for a symmetric equilibrium in the original game.

In sum, standard global game arguments guarantee the existence of a unique symmetric switching equilibrium in the original game. In addition, Lemma 4 tells us that there can be no other switching equilibria.

\section{Welfare}

In the benchmark model, concentration of trades in the $\mathrm{CN}$ is efficient whenever $\theta<\tilde{\theta}$. With uncertainty, the efficient threshold is different. The difference arises because (as shown in Subsection 2.3) a trader with liquidity preference $\tilde{\theta}$ should go to the CN only if all other traders do so. With dispersed preferences, a trader with preference $\tilde{\theta}$ must expect other traders to have a stronger preference for immediate execution and thus choose the DM. Hence, she should also go to the DM.

This argument holds for preferences close to $\tilde{\theta}$. The following proposition shows that total expected payoffs to all traders would be maximised if they could coordinate on trading at the CN up to a idiosyncratic liquidity preference $k^{*}$ that is smaller than, but $\nu$-close to, $\tilde{\theta}$. (Recall $F$ is the cumulative distribution function of the 


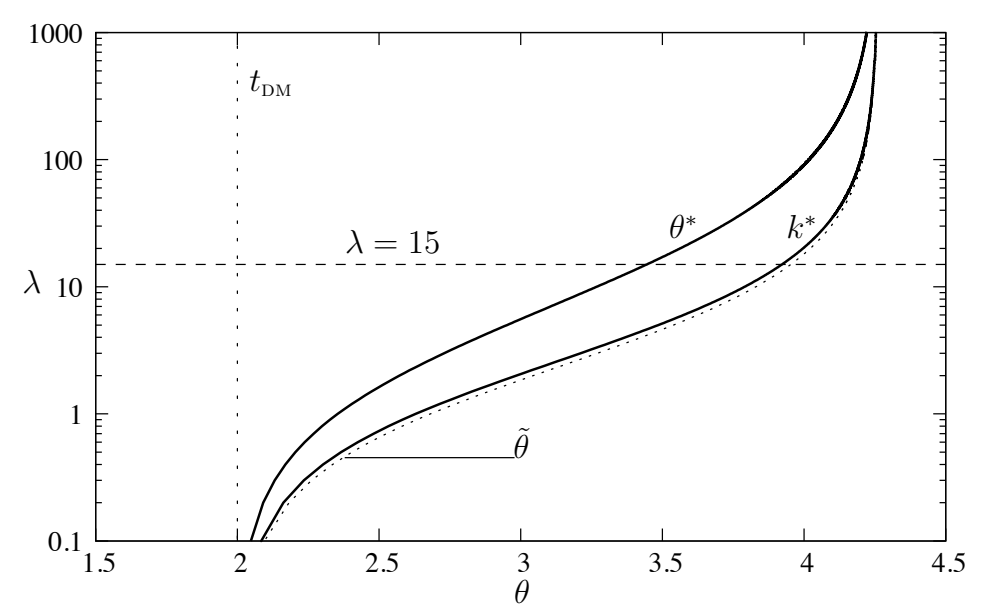

Results for $t_{\mathrm{CN}}=1, t_{\mathrm{DM}}=2, \nu=1$, and a truncated normal distribution with standard deviation $1 / 4$.

FiguRE 3. Equilibrium threshold $\theta^{*}$ versus efficient threshold $k^{*}$

idiosyncratic noise. The proof involves chiefly algebraic manipulation and is in the appendix).

Proposition 8. The strategy maximising expected total payoff to all traders in the private value game is $I_{k^{*}}$, where

$$
k^{*}=\tilde{\theta}-\nu \frac{\bar{\pi} / 2-K}{(1-\bar{\pi})}<\tilde{\theta}
$$

and $0 \leq K:=\int_{-1 / 2}^{1 / 2} F(z) \pi(F(z)) d z<\bar{\pi} / 2$.

If the equilibrium threshold $\theta^{*}$ is smaller than $k^{*}$, the equilibrium is inefficient. As our argument above relies on heterogeneous preferences, the deviation of $k^{*}$ from $\tilde{\theta}$ disappears for $\nu \rightarrow 0$, while the equilibrium threshold $\theta^{*}$ is independent from $\nu$ and bounded away from $\tilde{\theta}$. Note that $K$ depends on $F$, but not on $\nu$. So, for sufficiently small heterogeneity the equilibrium threshold is below the efficient one. The inefficiency arises because the decision to go to the $\mathrm{CN}$ increases expected payoffs also for other users of the CN due to network externalities, something that this is not accounted for by individual decisions.

Proposition 8 does not depend on the assumption of a geometric arrival process. But for a geometric distribution, the deviation of $k^{*}$ from $\tilde{\theta}$ can be shown once more to depend on the market liquidity $\lambda$. Figure 4 plots the efficient and the equilibrium threshold for different values of $\lambda$ when the idiosyncratic liquidity preferences are given by a (truncated) normal distribution and under a geometric arrival process process. For a uniform distribution of preferences, one can prove the equilibrium threshold is always below the efficient one. As figure 4 shows, this also tends to hold for other distributions and parameter values.

Although a large market does not guarantee order execution in the $\mathrm{CN}$, it nevertheless reduces the risk of mis-coordination between buyers and sellers. Hence, the 
thicker a market, the higher is the liquidity preference at which traders are indifferent in equilibrium. For $\lambda \rightarrow \infty$, the probability of order execution $\pi(\alpha)$ approaches $\ln (2)$ for all $\alpha>0$. From Propositions 5 and 8 we now readily deduce:

Corollary 9. For the geometric distribution of market size the thresholds $\theta^{*}, k^{*}$, and tipping point $\tilde{\theta}$ all converge to $\left(t_{\mathrm{DM}}-\ln (2) t_{\mathrm{CN}}\right) /(1-\ln (2))$ as $\lambda \rightarrow \infty$.

Proof. It suffices to note that $\lim _{\lambda \rightarrow \infty} K=\int_{-1 / 2}^{1 / 2} F(z) \bar{\pi} d z=\bar{\pi} / 2$, since the graph of $F$ is point-symmetric in $\left(0, \frac{1}{2}\right)$ due to the symmetry assumption on the distribution.

As we have argued above, the probability of order execution is bounded away from unity even for an arbitrarily large number of expected traders. For increasing $\lambda$, the equilibrium switching point $\theta^{*}$ (at which the heterogeneous traders are indifferent between the $\mathrm{CN}$ and the DM in equilibrium) and the efficient threshold $k^{*}$ (at which the DM is a dominating choice) are both increasing, and $\theta^{*}$ catches up with the efficient $k^{*}$.

This implies that for thick markets, the equilibrium of the private value game approaches efficiency. Although a thick market does not guarantee order execution in the $\mathrm{CN}$, it provides a sufficient incentive to coordinate on the $\mathrm{CN}$ whenever this is efficient. In the limit, the solution of the private value game also converges to the upper bound of the region with multiple equilibria in the benchmark game.

\section{Robustness}

We have argued that in a $\mathrm{CN}$, a larger volume of trades increases the probability of order execution, which leads to network externalities. Several other features of market intermediation lead to network externalities as well. In a traditional DM, the market maker faces inventory risk that declines with the volume of trade and thus allows her to lower trading costs and attract even more traders. In addition, the costs of price discovery and the risk of exploitation by insiders are decreasing in the proportion of orders that are directed to the same market venue. We now argue that these features reinforce the assumptions needed for achieving a unique switching equilibrium. We also discuss the robustness of our results to a situation where there are different kinds of traders, and to the uniform prior assumption.

\section{§5.1. Endogenous Trading Costs}

We assumed transaction fees at both markets to be set exogenously. In reality, costs to operate a CN are overhead and fees should be falling in the number of trades. Similarly, for a DM, the bid ask spread can be lower if more traders use this market. Reasons are, besides overhead costs, decreasing expected inventory per trade, lower costs of price discovery, and a lower risk of exploitation by insiders. As orders carry information used for price discovery, a dealer's bid-ask spread is generally decreasing 
in trading volume. These features strengthen the strategic complements driving our results on the allocation of order flow. To see this, suppose that $t_{\mathrm{CN}}(\alpha)$ is a decreasing function of the CN's market share $\alpha$ and $t_{\mathrm{DM}}(\alpha)$ is increasing in $\alpha$. The arguments in Section 3 concerning strategic complements carry through.

A more technical requirement for the argument in Section 3 is the existence of dominance regions. If $t_{\mathrm{CN}}(0)<t_{\mathrm{DM}}(1)$, the lower dominance region exists. This assumption can be justified by assuming that a CN cross-subsidises dry markets by revenues from markets in which it is already established or reduces fees below average costs in the introduction phase as an advertisement.

On the other hand, the DM will never charge an infinite fee, even if it has almost no customers. Traded assets have an inherent value and dealers may also use the CN to relieve themselves of involuntary inventory. Hence, the upper dominance region should exist as well.

\section{§5.2. Price Discovery}

Typically, electronic markets are first established as CNs and market orders are carried out at a price derived from traditional markets. Once established, many CNs expand by allowing limit orders and introduce their own price discovery. Then, they are classified as Electronic Communication Networks (ECNs). The submission of a limit order with a low limit greatly reduces the risk of an order not being executed. Therefore, going to an ECN would seem to become a dominant strategy for all traders, no matter how urgent their orders are, provided that transaction costs on the ECN are sufficiently low compared to the DM. However, price discovery done by dealers is more than just equalising supply and demand.

Dealers try to smooth prices over time by taking inventory. They also respond to indications of inside-trading. A low trading volume and uncertain prospects of the underlying asset are more likely leading to volatile prices in an ATS than in a DM. Traders who know the fair price may submit limit orders to an ECN but their orders might not be executed if the current price happens to be beyond their limit due to volatility. Hence, risk averse traders still have an incentive to choose the DM for trading such assets. At a DM, prices are more stable and orders are always executed.

Smoothing prices is a service that is valuable to customers, as is immediate order execution. DMs provide both services, while pure electronic markets can provide only one of the two. CNs provide smooth prices taken from the DM, electronic markets with price discovery provide almost certain order execution instead. For a trader, the trade-off between an ECN and a DM is similar to the trade-off between a CN and a DM. For traders with a low preference for the service of price-smoothing, the ECN is a dominant strategy, while traders with a high preference for smooth prices better choose the DM. Strategic complements arises from the fact that both market venues can reduce their price fluctuations with a higher turnover. Thus, the global 


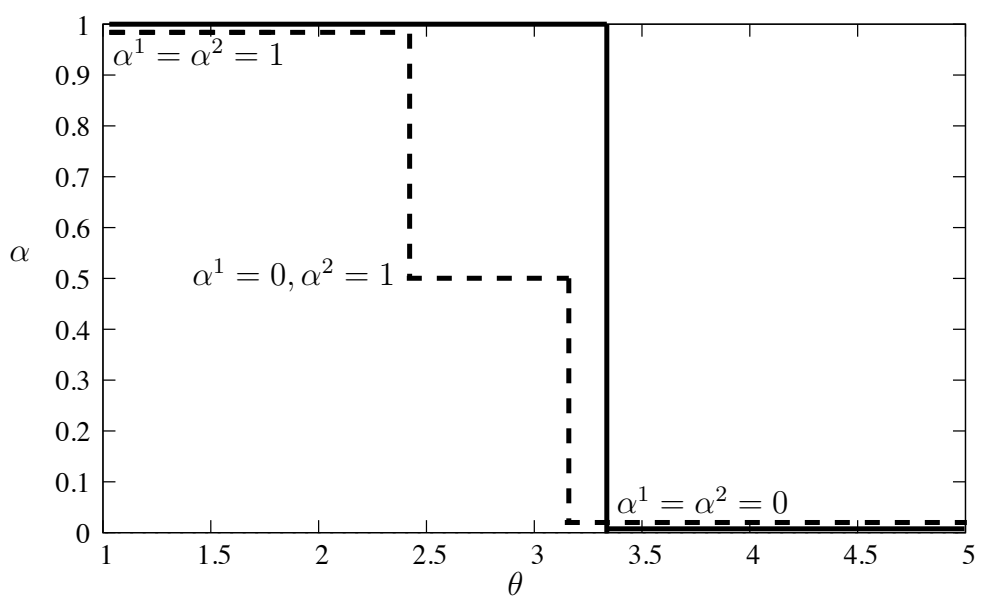

Results for $\lambda=15, t_{\mathrm{CN}}=1, t_{\mathrm{DM}}=2$, and $\xi_{1}=\xi_{2}=\frac{1}{2}$.

Solid line shows the switching equilibrium for $\delta_{\mathrm{DM}}^{1}=\frac{1}{10}$ and $\delta_{\mathrm{CN}}^{1}=\delta_{\mathrm{CN}}^{2}=\delta_{\mathrm{DM}}^{2}=0$. Both groups switch at $\theta \approx 3.32$.

Dashed line shows the switching equilibrium for $\delta_{\mathrm{DM}}^{1}=\frac{1}{2}$ and $\delta_{\mathrm{CN}}^{1}=\delta_{\mathrm{CN}}^{2}=\delta_{\mathrm{DM}}^{2}=0$. Group

1 traders switch at $\theta \approx 2.43$ and group 2 traders switch at $\theta \approx 3.14$.

FIGURE 4. Symmetric switching equilibrium with different kinds of traders

game approach can be applied in a similar way to the competition between an ECNs and DMs, though modelling the details will admittedly be more difficult.

These arguments also explain an empirical difference between the US and Europe. In the US, traditional markets operate as DMs and face competition by ATSs with price discovery, while in Europe, most stock exchanges are organised as auctions and do not take inventory. They face more competition from cheaper CNs (Degryse and Van Achter [8]).

\section{§5.3. Different kinds of traders}

Proposition 5 implies dealer markets may coexist with crossing networks when traders' liquidity preferences are sufficiently heterogeneous. In this subsection, we discuss a variant of this result that holds when there are other sources of heterogeneity among traders. For instance, some may be large institutional traders for whom revealing trades is costly and who value anonymity. That makes trading on a CN relatively more attractive for them.

Suppose there are $m>0$ groups of traders with relative proportions $\xi^{k} \in \mathbf{Q}_{+}$, $(k=1,2, \ldots, m)$ summing to unity. Trader $i$ from group $k$ has utility function:

$$
U_{k}\left(\theta_{i}, \alpha_{b}, \alpha_{s}\right)=\left(\theta_{i}-\delta_{\mathrm{CN}}^{k}-t_{\mathrm{CN}}\right) \pi^{e}\left(\theta_{i}, \alpha_{b}, \alpha_{s}\right)-\left(\theta_{i}-\delta_{\mathrm{DM}}^{k}-t_{\mathrm{DM}}\right),
$$

where $\delta_{\mathrm{CN}}^{k}$ and $\delta_{\mathrm{DM}}^{k}$ are group-specific parameters affecting a group's preferences over the crossing network and the dealer market, and $\theta_{i}$ is an idiosyncratic liquidity preference, as before. 
We have the following equilibrium existence result, as well as a characterisation result for small noise. For $G \subseteq\{1,2, \ldots, m\}$, define:

$$
P\left(\theta_{i}, G\right)=\Pi\left(\sum_{k \in G} \xi_{k}\right)-\sum_{k \in G} \xi_{k} \frac{\theta_{i}-\delta_{\mathrm{DM}}^{k}-t_{\mathrm{DM}}}{\theta_{i}-\delta_{\mathrm{CN}}^{k}-t_{\mathrm{CN}}},
$$

where $\Pi(\alpha)=\int_{0}^{\alpha} \pi(x) \mathrm{d} x$. Then the following holds: ${ }^{3}$

Proposition 10. This game has a unique symmetric switching equilibrium; as $\nu \rightarrow 0$, the groups of traders that go to the $C N$ are determined point-wise as $\arg \max _{G} P(\theta, G)$.

Thus, to find the (unique) symmetric threshold equilibrium, one may maximise $P$. We prove the proposition in the appendix, exploiting a known link between global games and the potential games of Monderer and Shapley [21]. Maximising $P$ is a relatively tractable problem in practice. For example, Figure 4 plots the switching equilibrium of a game with heterogeneous traders under two different assumptions for the group-specific parameters. Note how in the extended model with heterogeneous traders, the DM and CN may now coexist even if the noise scale $\nu$ that affects their liquidity preferences becomes small.

Furthermore, note that the expression for $\theta^{*}$ in Proposition 5 is a special case of the solution in stated in Proposition 10 with $m=\xi^{1}=1$ and $\delta_{\mathrm{CN}}^{1}=\delta_{\mathrm{DM}}^{1}=0$. In fact, the equilibria in the extended model share the property that the equilibrium thresholds are bounded away from $\tilde{\theta}$. Therefore, variants of the welfare results reported in Section 4 will also hold.

\section{§5.4. Non-Financial Markets}

Our results carry over to non-financial markets, provided that traders' size and the distribution of their liquidity preferences is the same on both sides of the market, and information is symmetric among all traders. Electronic matching markets can attract order flow away from traditional dealer markets, if they offer features that make them a dominant strategy for some traders on both sides of the market. The symmetry assumptions hold for some business to business markets (such as commodities markets) but not for markets in which larger intermediaries trade with smaller retail shops or for retailers dealing with consumers (such as amazon.com).

\section{$\S 5.5$. Non-uniform prior distributions}

Our results, particularly Proposition 5 on the uniqueness of the switching equilibrium for any $\nu>0$, assume that traders have a uniform prior on the support $\left[\theta_{L}, \theta_{H}\right]$ of the aggregate liquidity shock $\theta$. To conclude our paper, we briefly discuss the

\footnotetext{
${ }^{3}$ Unfortunately we have not been able to prove the counterpart of Lemma 4 for this setting. Thus, while a unique symmetric switching equilibrium exists, we are not sure that there are no other equilibria.
} 
robustness of our results with respect to this assumption, building on known results from the global games literature.

A sufficient condition for the existence of a switching equilibrium is that a higher liquidity preference leads a trader to shift up his posterior beliefs about the aggregate liquidity shock. Following Guimarães and Morris [17], say that the noise structure in the private value game satisfies first order stochastic dominance if, given a prior density $\phi$, the cumulative posterior distribution of a trader over $\theta$,

$$
H\left(\theta \mid \theta_{i}\right)=\int_{-\infty}^{\theta} \phi(x) f\left(\theta_{i}-x\right) \mathrm{d} x / \int_{-\infty}^{\infty} \phi(x) f\left(\theta_{i}-x\right) \mathrm{d} x,
$$

is decreasing in $\theta_{i}$ for each value of $\theta$. If this condition holds, the expected order execution probability (8), which may be written as

$$
\pi^{e}\left(\theta_{i}, \alpha_{b}, \alpha_{s}\right)=\int_{\theta} \pi\left(\alpha_{b}\left(\theta, a_{b}\right), \alpha_{s}\left(\theta, a_{s}\right)\right) \mathrm{d} H\left(\theta \mid \theta_{i}\right)
$$

is increasing for any switching strategy. Hence, the existence of a switching equilibrium may be established by arguments analogous to that in Section 3. Uniqueness is guaranteed with any prior if $\phi$ is smooth and the heterogeneity in idiosyncratic preferences, as measured by $\nu$, becomes small. Frankel, Morris and Pauzner [12] show that the noise structure of the game then converges to that of a game with a uniform prior. ${ }^{4}$

\section{Conclusion}

The emergence of alternative trading systems such as CNs increases inter-market competition for order flow. While increased competition may be welcomed from the perspective of market and price efficiency, the greater choice of trading venues fragments order flow and reduces liquidity and transparency, which are key to the functioning of financial markets. Hence, it is important to know under what conditions CNs may emerge as alternative trading systems. In this paper we presented a model building on the idea that the allocation of order flow between DMs and a CN may be understood as a game with network externalities.

The main achievement of our model is the removal of the multiplicity of equilibria in the allocation of order flow that has plagued previous models in the literature with similar features. We proved the existence of a unique switching equilibrium if traders have heterogeneous preferences about the value of immediate order execution. Our argument requires that traders face some uncertainty about their own positions in the distribution of everyone's preferences. That is key for applying global games techniques, but also more realistic than assuming common knowledge of the distribution of preferences amongst active traders.

\footnotetext{
${ }^{4}$ See Lemma A2 in [12]. This is a result solely about the noise structure of a global game, and applies to our game which has a similar noise structure, even though has payoff functions that do not satisfy the usual global game assumptions.
} 
In contrast to models with multiple equilibria, our analysis provides some definitive answers to the question under which conditions a $\mathrm{CN}$ can co-exist with a dealer market. For instance, if disutility from unexecuted orders arise due to price volatility, our model shows that orders for trading assets with low price volatility and large turnovers are initially submitted to a CN, while assets with high volatility or small volumes are exclusively traded on dealer markets. The main advantage of a CN are low transaction costs. Part of the service provided by a dealer may be understood as an insurance against buyer-seller mis-coordination. Therefore, traders with a preference for fast order execution prefer trading on a DM even at higher costs.

The properties of the equilibrium shed light on developments that redirect order flow away from traditional exchanges. A new trading venue could offer a service or a cost advantage that attracts some traders independent of the size of the new trading venue. Examples of services that have emerged in reality are after hour-trading, anonymity, and subsidised rerouting of orders on exchanges to ATS partners. This guarantees a lower bound on liquidity.

In Europe, CNs have been established mainly by brokers, who circumvent traditional exchanges by matching their customers' orders in-house at cheaper costs, if possible. They use traditional exchanges only for excess orders. In the US, traditional dealer markets are less automated and price discovery takes explicit account of potential orders by insiders. Here, ECNs have been the most successful alternative trading systems. Still, the allocation of order flow follows a similar pattern: traditional dealers provide a partial insurance against exploitation of liquidity traders by insiders, where transaction prices at ECNs do not provide this service. ${ }^{5}$ Liquidity traders may or may not find it worthwhile to pay a premium for this insurance, depending on their preferences.

By offering lower trading costs or special services that make a new market venue attractive for some customers even with low liquidity, a venue may create the minimal momentum required to attract further customers. If this is given, the new venue can gain an even larger market share, because as liquidity increases, the venue attracts more and more traders who would otherwise prefer the established intermediary.

\section{Appendix: Proofs}

Proof of Lemma 1. For a buyer on the CN, the number of additional buyers $k$ has a geometric distribution with $\mathbf{E}(k)=\alpha_{b} \lambda$. The probability of having $k$ other buyers on this market is

$$
p_{b}(k)=\frac{1}{1+\alpha_{b} \lambda}\left(\frac{\alpha_{b} \lambda}{1+\alpha_{b} \lambda}\right)^{k} .
$$

\footnotetext{
${ }^{5}$ Empirical evidence for this view is provided by Venkataraman [30].
} 
The probability of having $r$ sellers is

$$
p_{s}(r)=\frac{1}{1+\alpha_{s} \lambda}\left(\frac{\alpha_{s} \lambda}{1+\alpha_{s} \lambda}\right)^{r} .
$$

The probability of execution of a buyer's order, given that there are $k$ other buyers and $r$ sellers, is

$$
\pi_{b}(k, r)=\left\{\begin{array}{lll}
\frac{r}{k+1} & \text { if } & r \leq k \\
1 & \text { if } \quad r>k
\end{array}\right.
$$

The conditional probability of order execution, given that there are $k$ other buyers, is

$$
\begin{aligned}
\mathbf{E}\left(\pi_{b} \mid k\right) & =\sum_{r=0}^{k} \frac{r}{k+1} p_{s}(r)+1 \cdot p_{s}(r>k)=\sum_{r=0}^{k} \frac{r}{k+1} p_{s}(r)+1-\sum_{r=0}^{k} p_{s}(r) \\
& =1-\sum_{r=0}^{k}\left(1-\frac{r}{k+1}\right) p_{s}(r)=1-\frac{1}{1+\alpha_{s} \lambda}\left[\sum_{r=0}^{k} q_{s}^{r}-\frac{1}{k+1} \sum_{r=0}^{k} r q_{s}^{r}\right],
\end{aligned}
$$

where $q_{s}:=\frac{\alpha_{s} \lambda}{1+\alpha_{s} \lambda}$. Using

$$
1-q_{s}=\frac{1}{1+\alpha_{s} \lambda}, \quad \sum_{r=0}^{k} q^{r}=\frac{1-q^{k+1}}{1-q} \quad \text { and } \quad \sum_{r=0}^{k} r q^{r}=\frac{q\left[1-q^{k}(k+1-q k)\right]}{(1-q)^{2}}
$$

we find that

$$
\begin{aligned}
\mathbf{E}\left(\pi_{b} \mid k\right) & =q_{s}^{k+1}+\frac{\alpha_{s} \lambda}{k+1}\left[1-q_{s}^{k}\left(k+1-q_{s} k\right)\right]=q_{s}^{k+1}+\alpha_{s} \lambda\left[\frac{1-q_{s}^{k+1}}{k+1}-q_{s}^{k}+q_{s}^{k+1}\right] \\
& =q_{s}^{k+1}\left(1+\alpha_{s} \lambda\right)+\alpha_{s} \lambda\left[\frac{1-q_{s}^{k+1}}{k+1}-q_{s}^{k}\right] .
\end{aligned}
$$

The probability of order execution is

$$
\begin{aligned}
\mathbf{E}\left(\pi_{b}\right) & =\sum_{k=0}^{\infty} \mathbf{E}\left(\pi_{b} \mid k\right) p_{b}(k) \\
& =\sum_{k=0}^{\infty}\left[q_{s}^{k+1}\left(1+\alpha_{s} \lambda\right)+\alpha_{s} \lambda\left(\frac{1-q_{s}^{k+1}}{k+1}-q_{s}^{k}\right)\right] \frac{1}{1+\alpha_{b} \lambda} q_{b}^{k}
\end{aligned}
$$

where $q_{b}:=\frac{\alpha_{b} \lambda}{1+\alpha_{b} \lambda}$. This equals

$$
\begin{aligned}
& \frac{1+\alpha_{s} \lambda}{1+\alpha_{b} \lambda} \sum_{k=0}^{\infty} q_{s}^{k+1} q_{b}^{k}+\frac{\alpha_{s} \lambda}{1+\alpha_{b} \lambda} \sum_{k=0}^{\infty}\left(\frac{q_{b}^{k}}{k+1}-\frac{q_{s}^{k+1} q_{b}^{k}}{k+1}-q_{s}^{k} q_{b}^{k}\right) \\
& =\frac{1+\alpha_{s} \lambda}{1+\alpha_{b} \lambda} q_{s} \sum_{k=0}^{\infty} q_{s}^{k} q_{b}^{k}+\frac{\alpha_{s} \lambda}{1+\alpha_{b} \lambda}\left[\frac{1}{q_{b}} \sum_{k=1}^{\infty} \frac{q_{b}^{k}-q_{s}^{k} q_{b}^{k}}{k}-\sum_{k=0}^{\infty} q_{s}^{k} q_{b}^{k}\right] \\
& =\frac{\alpha_{s}}{\alpha_{b}} \sum_{k=1}^{\infty} \frac{q_{b}^{k}-q_{s}^{k} q_{b}^{k}}{k} .
\end{aligned}
$$

Using $\sum_{k=1}^{\infty} q^{k} / k=-\ln (1-q)$, we find that

$$
\mathbf{E}\left(\pi_{b}\right)=\frac{\alpha_{s}}{\alpha_{b}}\left[\ln \left(1-q_{b} q_{s}\right)-\ln \left(1-q_{b}\right)\right]=\frac{\alpha_{s}}{\alpha_{b}} \ln \frac{1+\left(\alpha_{s}+\alpha_{b}\right) \lambda}{1+\alpha_{s} \lambda}
$$




$$
=\frac{\alpha_{s}}{\alpha_{b}} \ln \left(1+\frac{\alpha_{b} \lambda}{1+\alpha_{s} \lambda}\right) .
$$

The execution probability for sell orders is calculated by interchanging the subscripts $b$ and $s$.

Proof of Proposition 8. Given a switching strategy around $k$, the unconditional expected payoff is given by

$$
\int_{-\frac{1}{2}}^{\frac{1}{2}} \int_{\theta_{L}}^{k-\nu u_{i}} \pi\left(\alpha_{s}\left(\theta, I_{k}\right)\right)\left(\theta+\nu u_{i}-t_{\mathrm{CN}}\right) \mathrm{d} \theta+\int_{k-\nu u_{i}}^{\theta_{H}} \theta+\nu u_{i}-t_{\mathrm{DM}} \mathrm{d} \theta \mathrm{d} F\left(u_{i}\right) .
$$

Exploiting that $u_{i}$ is distributed in $\left[-\frac{1}{2},+\frac{1}{2}\right]$, this expression may be rewritten as

$$
\begin{gathered}
\int_{-\frac{1}{2}}^{\frac{1}{2}} \int_{\theta_{L}}^{k-\frac{\nu}{2}} \bar{\pi} \cdot\left(\theta+\nu u_{i}-t_{\mathrm{CN}}\right) \mathrm{d} \theta+\int_{k-\nu u_{i}}^{\theta_{H}} \theta+\nu u_{i}-t_{\mathrm{DM}} \mathrm{d} \theta \\
\quad+\int_{k-\frac{\nu}{2}}^{k-\nu u_{i}} \pi\left(\alpha_{s}\left(\theta, I_{k}\right)\right)\left(\theta+\nu u_{i}-t_{\mathrm{CN}}\right) \mathrm{d} \theta \mathrm{d} F\left(u_{i}\right) \\
=\int_{-\frac{1}{2}}^{\frac{1}{2}} \int_{\theta_{L}}^{k-\nu \frac{1}{2}} \bar{\pi} \cdot\left(\theta+\nu u_{i}-t_{\mathrm{CN}}\right) \mathrm{d} \theta+\int_{k-\nu u_{i}}^{\theta_{H}} \theta+\nu u_{i}-t_{\mathrm{DM}} \mathrm{d} \theta \\
\quad+\int_{-\frac{\nu}{2}}^{-\nu u_{i}} \pi\left(F\left(-\frac{z}{\nu}\right)\right)\left(z+k+\nu u_{i}-t_{\mathrm{CN}}\right) \mathrm{d} z \mathrm{~d} F\left(u_{i}\right) .
\end{gathered}
$$

To set up a first order condition, we differentiate under the integral sign to get

$$
\begin{aligned}
& \int_{-\frac{1}{2}}^{\frac{1}{2}} \bar{\pi} \cdot\left(k-\frac{\nu}{2}+\nu u_{i}-t_{\mathrm{CN}}\right)-\left(k-t_{\mathrm{DM}}\right)+\int_{-\frac{\nu}{2}}^{-\nu u_{i}} \pi\left(F\left(-\frac{z}{\nu}\right)\right) \mathrm{d} z \mathrm{~d} F\left(u_{i}\right) \\
= & (\bar{\pi}-1) k+t_{\mathrm{DM}}-\bar{\pi} t_{\mathrm{CN}}-\frac{1}{2} \nu \bar{\pi}+\int_{-\frac{1}{2}}^{\frac{1}{2}} \int_{-\frac{\nu}{2}}^{-\nu F^{-1} F\left(u_{i}\right)} \pi\left(F\left(-\frac{z}{\nu}\right)\right) \mathrm{d} z f\left(u_{i}\right) \mathrm{d} u_{i} \\
= & (\bar{\pi}-1) k+t_{\mathrm{DM}}-\bar{\pi} t_{\mathrm{CN}}-\frac{1}{2} \nu \bar{\pi}+\int_{0}^{1} \int_{-\frac{\nu}{2}}^{-\nu F^{-1}(x)} \pi\left(F\left(-\frac{z}{\nu}\right)\right) \mathrm{d} z \mathrm{~d} x \\
= & (\bar{\pi}-1) k+t_{\mathrm{DM}}-\bar{\pi} t_{\mathrm{CN}}-\frac{1}{2} \nu \bar{\pi}+\nu \int_{0}^{1} \int_{F^{-1}(x)}^{\frac{1}{2}} \pi(F(z)) \mathrm{d} z \mathrm{~d} x,
\end{aligned}
$$

where we have used changes of variables in the last two lines. Solve for a zero to find the switching point that maximises expected payoff:

$$
k^{*}=\frac{t_{\mathrm{DM}}-\bar{\pi} t_{\mathrm{CN}}}{1-\bar{\pi}}-\frac{\nu}{2(1-\bar{\pi})}\left[\bar{\pi}-2 \int_{0}^{1} \int_{F^{-1}(x)}^{\frac{1}{2}} \pi(F(z)) \mathrm{d} z \mathrm{~d} x\right] .
$$

By Fubini's theorem, we may change the order of integration for the double integraldenoted $K$ in the statement of our proposition - so that

$$
\iint_{D} \pi(F(z)) \mathrm{d} z \mathrm{~d} x=\iint_{D} \pi(F(z)) \mathrm{d} x \mathrm{~d} z=\int_{-\frac{1}{2}}^{\frac{1}{2}} \int_{0}^{F(z)} \pi(F(z)) \mathrm{d} x \mathrm{~d} z,
$$


where we used that the two-dimensional domain of integration can be written as

$$
\begin{aligned}
D & :=\left\{(x, z) \in \mathbf{R}^{2} \mid 0 \leq x \leq 1 \text { and }-\frac{1}{2} \leq z \leq \frac{1}{2} \text { and } z \geq F^{-1}(x)\right\} \\
& =\left\{(x, z) \in \mathbf{R}^{2} \mid 0 \leq x \leq 1 \text { and }-\frac{1}{2} \leq z \leq \frac{1}{2} \text { and } x \leq F(z)\right\} .
\end{aligned}
$$

Integrating the inner integral gives $K=\int_{-\frac{1}{2}}^{\frac{1}{2}} F(z) \pi(F(z)) \mathrm{d} z$. Finally, to see that the inequality on $K$ in the proposition holds, note that $\pi(\alpha)<\bar{\pi}$ for almost all $\alpha$. Substituting $\bar{\pi}$ for $\pi(\cdot)$, we find $K<\bar{\pi} \int_{-\frac{1}{2}}^{\frac{1}{2}} F(z) \mathrm{d} z=\frac{1}{2} \bar{\pi}$, where the fact that the integral evaluates to $\frac{1}{2}$ follows from the point symmetry of the graph of $F$ in the point $\left(0, \frac{1}{2}\right)$.

Proof of Proposition 10. As in Subsection 3.4, consider a modified game, $\Gamma$, in which agents choose to go to the $\mathrm{CN}$ or the DM before they learn whether they will want to buy or sell the asset, so that strategies of buyers and sellers are identical. Formally, let $a^{k}$ be the proportion of buyers and sellers of group $k$ that go to the $\mathrm{CN}$ when their liquidity preference is $\theta_{i}$. Then $\alpha_{b}^{k}(\theta)=\alpha_{s}^{k}(\theta):=\alpha^{k}(\theta)$ for all $k$ and all $\theta$. For the purpose of this proof, we will relabel the liquidity preferences by defining $\eta_{i}=-\theta_{i}$; nothing substantial hinges on this labelling. ${ }^{6}$ Denoting the aggregate proportion of traders going to the $\mathrm{CN}$ by $\alpha$, payoffs for group $k$-traders are then given by

$$
U_{k}\left(\eta_{i}, \alpha\right)=\left(\eta_{i}+\delta_{k}+t_{\mathrm{DM}}\right)-\left(\eta_{i}+\delta_{k}+t_{\mathrm{CN}}\right) \pi^{e}\left(-\eta_{i}, \alpha, \alpha\right)
$$

By Lemma A1 in Frankel, Morris and Pauzner [12], for any $\nu$, this modified game has an essentially unique, monotonic switching equilibrium. In this equilibrium, for each $\eta$, all traders in the same group choose the same venue: $a^{k}(\theta) \in\{0,1\}$ for all $k$ and all $\theta$. We may therefore describe the equilibrium $a^{\nu}(\eta):=\left(a^{1}(\eta), \ldots, a^{m}(\eta)\right)$ as a profile of step functions indicating the proportion ( 0 or 1$)$ of traders that go to the $\mathrm{CN}$ for each value of $\eta$. By virtue of our relabelling of $\theta$, each $a^{k}(\eta)$ is an increasing function; for fixed $\eta$, the profile $a^{\nu}(\eta)$ gives a coordinate (in fact, a corner) in the $m$-dimensional unit cube. Following the argument in Subsection 3.4, this equilibrium is the sole symmetric switching equilibrium of the generalised game with different groups of traders. This proves the first part of the proposition.

We will characterise the limiting switching equilibrium profile $\bar{a}$ obtained when $\nu \rightarrow 0$. Identify the subsets $G \subseteq\{1,2, \ldots, m\}$ with the corners of the $m$-dimensional unit cube and extend $P$ as follows to the unit cube:

$$
\tilde{P}\left(\eta, \alpha^{1}, \ldots, \alpha^{m}\right)=\int_{0}^{\sum_{k \in G} \xi_{k} \alpha_{k}} \pi(x) d x-\sum_{k \in G} \xi_{k} \alpha_{k} \frac{\eta+\delta_{\mathrm{DM}}^{k}+t_{\mathrm{DM}}}{\eta+\delta_{\mathrm{CN}}^{k}+t_{\mathrm{CN}}} .
$$

As a key step, we will approximate the modified game using a series of finite player games. Let $\ell$ be the least common denominator of $\xi_{1}, \ldots, \xi_{m}$. For each $n \in \mathbf{N}$, let $\Gamma^{n}$ be the finite $\ell n$-player global game with payoff functions as in (13). Since the number

\footnotetext{
${ }^{6}$ The relabelling is convenient, because it allows us to apply standard global game results that deal with monotonically increasing strategy profiles.
} 
of players is a multiple of $\ell$, the fractions $\alpha$ and $\alpha_{1}, \ldots, \alpha_{k}$ are still well defined for any action profile and any $\eta$. Again by Lemma A1 in [12], for each $\nu>0$, each game $\Gamma^{n}$ has an essentially unique, increasing switching equilibrium $a^{n \nu}$, in which for each $\eta$, all traders in the same group choose the same venue. Thus, each $a^{n \nu}(\eta)$ must be a corner of the $m$-dimensional unit cube. We now justify our approximation of $\Gamma$ by the games $\Gamma^{n}$ by proving the following claim. It states that, for any $\nu>0$, the equilibria $a^{n \nu}$ of the games $\Gamma^{n}$ converge to the equilibrium $a^{\nu}$ of $\Gamma$.

Claim 1. For each $\nu>0, a^{n \nu} \rightarrow a^{\nu}$ pointwise as $n \rightarrow \infty$.

Fix some $\nu>0$ and consider the sequence of equilibria $\left\{a^{n \nu}\right\}_{n \in \mathbf{N}}$. As the ("Helly") space of increasing functions is sequentially compact (see [27], example 107), we may assume this sequence converges to a limit $\bar{a}^{\nu}$. By the dominated convergence theorem, expectations under the utility functions $U_{k}$ converge along the sequence $\left\{a^{n \nu}\right\}_{n \in \mathbf{N}}$, so for each $\eta, \bar{a}^{\nu}(\eta)$ is a joint best reply to $\bar{a}^{\nu}$. Therefore $\bar{a}^{\nu}$ is an equilibrium of $\Gamma$. By the essential uniqueness of $a^{\nu}$, we must have $a^{\nu}=\bar{a}^{\nu}$, proving our claim.

Next, note that, for fixed $\eta$, the derived complete information game given by payoffs

$$
U_{k}^{C}(\eta, \alpha):=\left(\eta+\delta_{k}+t_{\mathrm{DM}}\right)-\left(\eta+\delta+t_{\mathrm{CN}}\right) \pi(\alpha)
$$

is a potential game with potential function

$$
\tilde{P}^{n}\left(\eta, \alpha_{1}, \ldots, \alpha_{k}\right):=\sum_{x=0}^{\ell n \sum \xi_{k} \alpha_{k}} \frac{\pi(x /(\ell n))}{\ell n}-\sum_{k} \xi_{k} \alpha_{k} \frac{\eta+\delta_{\mathrm{DM}}^{k}+t_{\mathrm{DM}}}{\eta+\delta_{\mathrm{CN}}^{k}+t_{\mathrm{CN}}} .
$$

By Theorem 4 in [12], if (for fixed $\eta$ ) the action profile $\underline{a}^{n} \in \arg \max \tilde{P}^{n}\left(\eta, \alpha_{1}, \ldots, \alpha_{k}\right)$, then $a^{n \nu}(\eta)=\underline{a}^{n}$ for sufficiently small $\nu$. We claim that $\tilde{P}^{n}$ relates to $\tilde{P}$ as follows:

Claim 2. For large $n, \arg \max \tilde{P}^{n}\left(\eta, \alpha_{1}, \ldots, \alpha_{k}\right) \subseteq \arg \max \tilde{P}\left(\eta, \alpha_{1}, \ldots, \alpha_{k}\right)$.

Consider the sequence $\left\{\underline{a}^{n}\right\}$. Without loss of generality we may assume this sequence converges to some $a^{*}$. We claim that $a^{*} \in \arg \max \tilde{P}\left(\eta, \alpha_{1}, \ldots, \alpha_{k}\right)$. For suppose this is not the case. Then there is $\alpha^{* *}$ such that $\tilde{P}\left(\eta, \alpha^{* *}\right)>\tilde{P}\left(\eta, a^{*}\right)+d$ and consequently, by the continuity of $\tilde{P}$, there are neighbourhoods $\mathcal{N}^{*}$ of $a^{*}$ and $\mathcal{N}^{* *}$ of $\alpha^{* *}$ such that $\tilde{P}(\eta, x)-d / 4>\tilde{P}(\eta, y)+d / 4$ for each $x \in \mathcal{N}^{* *}$ and $y \in \mathcal{N}^{*}$. Since our assumptions on $\pi$ imply it is Riemann integrable, for each point $z, \tilde{P}^{n}(\eta, z) \rightarrow \tilde{P}(\eta, z)$ as $n \rightarrow \infty$ (by the definition of the Riemann integral). We may choose $n$ sufficiently large so that the following holds: $\underline{a}^{n} \in \mathcal{N}^{*}$; there is at least one point $z$ of the action space of $\Gamma^{n}$ in $\mathcal{N}^{* *}$; and $\tilde{P}^{n}(\eta, z)$ and $\tilde{P}^{n}\left(\eta, \underline{a}^{n}\right)$ are within $d / 4$ of $\tilde{P}(\eta, z)$ and $\tilde{P}\left(\eta, a^{*}\right)$. Then

$$
\tilde{P}^{n}\left(\eta, \underline{a}^{n}\right) \geq \tilde{P}^{n}(\eta, z)>\tilde{P}(\eta, z)-d / 4>\tilde{P}\left(\eta, a^{*}\right)+(d / 4)>\tilde{P}^{n}\left(\eta, \underline{a}^{n}\right),
$$

but that is a contradiction. This proves our claim.

We are now ready to characterise the limiting switching equilibrium profile $\bar{a}$ of the game $\Gamma$, obtained when $\nu \rightarrow 0$. It suffices to characterise $\bar{a}$ at all but finitely many points. Let $D_{0}$ be the (finite) set of discontinuities of $\bar{a}, D_{1}$ be the (finite) set of 
points at which $\max P(\eta, \cdot)$ (which is increasing in $\eta$ ) is not uniquely defined, and $D=D_{0} \cup D_{1}$. Fix some $\eta^{*} \notin D$. We will prove the proposition by showing that $\bar{a}\left(\eta^{*}\right) \in \arg \max \tilde{P}\left(\eta^{*}, \alpha^{1}, \ldots, \alpha^{m}\right)$. By our claims above, we know that for some $a^{*}$ :

$$
\lim _{n \rightarrow \infty} \lim _{\nu \rightarrow 0} a^{\nu n}\left(\eta^{*}\right)=a^{*} \in \arg \max P \quad \text { and } \quad \lim _{n \rightarrow \infty} a^{\nu n}\left(\eta^{*}\right)=a^{\nu}\left(\eta^{*}\right) .
$$

Note that the proposition is proved if we may swap the double limit in (14), so that

$$
\lim _{\nu \rightarrow 0} a^{\nu}\left(\eta^{*}\right)=\lim _{\nu \rightarrow 0} \lim _{n \rightarrow \infty} a^{n \nu}\left(\eta^{*}\right)=\lim _{n \rightarrow \infty} \lim _{\nu \rightarrow 0} a^{n \nu}\left(\eta^{*}\right)=a^{*} \in \arg \max P .
$$

By the Moore-Osgood theorem, a sufficient condition is that the first limit in (14) converges uniformly. Thus we will show:

Claim 3. There are $\underline{n} \in \mathbf{N}$ and $\underline{\nu}>0$ such that for all $n>\underline{n}$ and all $0<\nu \leq \underline{\nu}$, we have $a^{n \nu}\left(\eta^{*}\right)=\underline{a}^{n}$, where $\underline{a}^{n}$ is the greatest element in $\arg \max \tilde{P}^{n}\left(\eta^{*}, \alpha_{1}, \ldots, \alpha_{k}\right)$. We will prove this using methods in Basteck, Daniëls and Heinemann [2].

Since, for each $\nu, a^{\nu}(\eta)$ converges to $\bar{a}(\eta)$ for each $\eta$, and for each $\nu, a^{\nu}$ is a profile of increasing functions taking on only finitely many values, there is some $\underline{\nu}>0$ such that, for all $0<\nu \leq \underline{\nu}$, and all $\eta$ in a 8$\underline{\nu} m$-neighbourhood of $\eta^{*}, a^{\nu}(\eta)=\bar{a}\left(\eta^{*}\right)$. Similarly, we may choose $\underline{n}$ such that $\underline{a}^{n}\left(\eta^{*}-4 \underline{\nu} m\right)=\underline{a}^{n}\left(\eta^{*}+4 \underline{\nu} m\right)$ and constant in-between. Now fix some $n$ and some $\nu$ satisfying the hypothesis of our claim. Given some fixed $\eta^{* *}$ that we will choose below, introduce the binary action game e with the same players as $\Gamma$, but payoffs ${ }^{7}$

$$
\tilde{U}_{k}\left(1, \alpha, x_{i}\right):=\left\{\begin{array}{ll}
-1 & \text { if } x_{i}<\eta^{* *}, \\
U_{k}\left(\alpha, \eta^{* *}\right) & \text { if } x_{i} \geq \eta^{* *} .
\end{array},\right.
$$

and $\tilde{U}_{k}\left(0, \alpha, x_{i}\right)=0$ (we identify action 1 with going to the $\mathrm{CN}$ and action 0 with going to the DM). Compared with the global game $\Gamma^{n}$, the distribution of individual signals remains unchanged. We assume an improper uniform prior, so the posterior beliefs in e are well defined and take the same form as in [2] and in $\Gamma^{n}$.

Now suppose $\underline{a}^{n}>a^{n \nu}\left(\eta^{*}\right)$ (in the pointwise ordering). Set $\eta^{* *}=\eta-2 \nu m$. By Theorem 2 in [2], in the game $\mathbf{e}$, there exists an equilibrium strategy profile $y$ such that $y(x)=\underline{a}^{n}$ for $x \geq \eta^{*}-\nu m$. Now consider the strategy profile $a=\max \left(a^{n \nu}, y\right)$. Due to strategic complements and the fact that $U_{k}$ is monotonic in $\eta$, an upper best reply iteration in $\Gamma^{n}$ starting from $a$ must lead upwards and converge to some equilibrium of $\Gamma$. Moreover, $a(\eta) \geq \underline{a}^{n}>a^{n \nu}\left(\eta^{*}\right)$ for $\eta>\eta^{*}-\nu m$. Yet this contradicts the uniqueness of the equilibrium $a^{n \nu}$ in $\Gamma^{n}$. So conclude $\underline{a}^{n} \ngtr a^{n \nu}\left(\eta^{*}\right)$.

Next, we prove $\underline{a}^{n} \geq a^{n \nu}\left(\eta^{*}\right)$. Consider $a^{n \nu}$. We know this equilibrium profile is increasing, so (since is has at most $m$ jumps), $3 \nu m$-near to $\eta^{*}$ there must be some point $\eta^{* *}>\eta^{*}$ such that $a^{n \nu}$ is constant in a $2 \nu m$ neighbourhood of $\eta^{* *}$. We will shift $a^{v n}$ to the right by $\Delta=\eta^{* *}-\eta^{*}$, bound the resulting strategy profile by $a^{\nu n}\left(\eta^{* *}\right)$ and consider it as a strategy profile in e. To this end, let $y(x)=\min \left\{a^{n \nu}(x-\Delta), a^{n \nu}\left(\eta^{* *}\right)\right\}$.

\footnotetext{
${ }^{7}$ We use a slight relabelling of e compared with Basteck et al. [2], who take $\eta^{*}=0$ and $\nu=1$.
} 
Due to strategic complements, and $\tilde{U}_{k}$ being monotonic in $\theta$, a best reply iteration in e starting from $y$ must lead upwards and converge to some equilibrium where $y(x) \geq a^{n \nu}\left(\eta^{* *}\right)$ for some $x$. By Theorem 2 in [2], this implies $\underline{a}^{n} \geq a^{n \nu}\left(\eta^{* *}\right)$, and we know $a^{n \nu}\left(\eta^{* *}\right) \geq a^{n \nu}\left(\eta^{*}\right)$. Thus, our claim is proved.

\section{References}

[1] Advancedtrading.com article: Bats exchange offers rebate for removing liquidity via dark scan.

[2] Basteck, C., Daniëls, T. R., and Heinemann, F. Characterising equilibrium selection in global games with strategic complementarities. Revised version of SFB working paper. Available at http://www.macroeconomics.tu-berlin.de/fileadmin/fg124 /Basteck/Characterising-new.pdf, 2010.

[3] Carlsson, H., And van Damme, E. Global games and equilibrium selection. Econometrica 61, 5 (1993), 989-1018.

[4] Chowdhry, B., And Nanda, V. Multimarket trading and market liquidity. Review of Financial Studies, 3 (1991), 483-511.

[5] Conrad, J., Johnson, K., and Wahal, S. Institutional trading and alternative trading systems. Journal of Financial Economics 70 (2003), 99-134.

[6] Daniëls, T., Jager, H., and Klaassen, F. Currency crises with the threat of an interest rate defence. Journal of International Economics (2011).

[7] Degryse, H. Competition between financial markets in europe: What can be expected from MiFID? Financial Markets and Portfolio Management 23 (2009), 93-103.

[8] Degryse, H., And Achter, M. V. Alternative trading systems and liquidity. In Technology and Finance: Challenges, Business Strategies and Policymakers, M. Balling, F. Lierman, and A. Mullineux, Eds. Routledge: London and New York, 2002, ch. 10.

[9] Degryse, H., Achter, M. V., And Wuyts, G. Dynamic order submission strategies with competition between a dealer market and a crossing network. Journal of Financial Economics 91 (2009), 319-338.

[10] Degryse, H., van Achter, M., And Wuyts, G. Shedding light on dark liquidity pools. TILEC Discussion paper 2008-039, 2008.

[11] Ellison, G., and Fudenberg, D. Knife-edge or plateau: When do market models tip? Quarterly Journal of Economics, 118 (2003), 1249-78.

[12] Frankel, D. M., Morris, S., And Pauzner, A. Equilibrium selection in global games with strategic complementarities. Journal of Economic Theory 108, 1 (2003), 1-44.

[13] GeHrig, T. Intermediation in search markets. Journal of Economics and Management Strategy 2 (1993), 97-120.

[14] Glosten, L. Is the electronic order book inevitable? Journal of Finance 49 (1994), 1127-1161.

[15] Goldstein, I., And Pauzner, A. Demand-deposit contracts and the probability of bank runs. Journal of Finance 60, 3 (2005), 1293-327.

[16] Gresse, C. The effect of crossing-network trading on dealer market's bid-ask spreads. European Financial Management 12 (2006), 143-160.

[17] Guimaraes, B., And Morris, S. Risk and wealth in a model of self-fulfilling currency attacks. Journal of Monetary Economics 54, 8 (2007), 2205-30.

[18] Hendershott, T., And Mendelson, H. Crossing networks and dealer markets: Competition and performance. The Journal of Finance 55, 5 (2000), 2071-2115. 
[19] Karp, L., Lee, I. H., And Mason, R. A global game with strategic substitutes and complements. Games and economic behaviour 60 (2007), 155-175.

[20] Mao YE. Non-execution and market share of crossing networks. Mimeo, 2010.

[21] Monderer, D., And Shapley, L. S. Potential games. Games and Economic Behavior 14, 1 (1996), 124-43.

[22] Morris, S., And Shin, H. S. Global games: Theory and applications. In Advances in Economics and Econometrics: Eight World Congress, M. Dewatripont, L. P. Hansen, and S. J. Turnovsky, Eds. Cambridge: Cambridge University Press, 2003, pp. 56-114.

[23] Morris, S., And Shin, H. S. Heterogeneity and uniqueness in interaction games. In The Economy as an Evolving Complex System, L. E. Blume and S. N. Durlauf, Eds., vol. III. 2006, pp. 207-242.

[24] Myerson, R. B. Population uncertainty and poisson games. International Journal of Game Theory 27, 3 (1998), 375-92.

[25] Pagano, M. Trading volume and asset liquidity. Quarterly Journal of Economics 104 (1989), 255-274.

[26] Parlour, C. A., And Seppi, D. J. Liquidity-based competition for order flow. Review of Financial Studies 16, 301-343 (2003).

[27] Steen, L., And Seebach, J. Counterexamples in Topology. Mineola, New York: Dover Publications Inc, 1995.

[28] Stoll, H. R. Electronic trading in stock markets. Journal of Economic Perspectives 20 (2006), $153-174$.

[29] Theissen, E. Floor versus screen trading: Evidence from the german stock market. Journal of Institutional and Theoretical Economics 158 (2002), 32-54.

[30] Venkataraman, K. Automated versus floor trading: An analysis of execution costs on the paris and new york exchanges. Journal of Finance 61 (2001), 1445-1485.

[31] Viswanathan, S., AND Wang, J. J. Market architecture: Limit-order books versus dealership markets. Journal of Financial Markets 5 (2002), 127-167. 


\section{SFB 649 Discussion Paper Series 2013}

For a complete list of Discussion Papers published by the SFB 649, please visit http://sfb649. wiwi.hu-berlin.de.

001 "Functional Data Analysis of Generalized Quantile Regressions" by Mengmeng Guo, Lhan Zhou, Jianhua Z. Huang and Wolfgang Karl Härdle, January 2013.

002 "Statistical properties and stability of ratings in a subset of US firms" by Alexander B. Matthies, January 2013.

003 "Empirical Research on Corporate Credit-Ratings: A Literature Review" by Alexander B. Matthies, January 2013.

004 "Preference for Randomization: Empirical and Experimental Evidence" by Nadja Dwenger, Dorothea Kübler and Georg Weizsäcker, January 2013.

005 "Pricing Rainfall Derivatives at the CME" by Brenda López Cabrera, Martin Odening and Matthias Ritter, January 2013.

006 "Inference for Multi-Dimensional High-Frequency Data: Equivalence of Methods, Central Limit Theorems, and an Application to Conditional Independence Testing" by Markus Bibinger and Per A. Mykland, January 2013.

007 "Crossing Network versus Dealer Market: Unique Equilibrium in the Allocation of Order Flow" by Jutta Dönges, Frank Heinemann and Tijmen R. Daniëls, January 2013. 